\title{
Optimization of Energy-Efficient Speed Profile for Electrified Vehicles
}

by

Hadi Abbas

A thesis submitted in partial fulfillment of the requirements for the degree of Master of Science in Engineering (Automotive Systems Engineering) in the University of Michigan-Dearborn 2018

Master's Thesis Committee:

Assistant Professor Youngki Kim, Chair Assistant Professor Stanley Baek

Professor Taehyun Shim 


\section{Dedication}

This thesis work is dedicated to my family, who have made me the man I am today and have provided me with all the love and support. They have always been great examples and have taught me to work hard for the things I aspire to achieve. This work is also dedicated to Moon Diab, my constant source of support, love, and encouragement during the challenges of graduate school, life, and the great changes that happened in my life. This work is also dedicated to my friend, professor, and advisor, Youngki Kim, who has taught me so much and has given me an unbelievable opportunity throughout graduate school. 


\section{Acknowledgements}

I would first like to thank my thesis advisor Assistant Professor Youngki Kim of the College of Engineering and Computer Science at the University of Michigan in Dearborn. The door to Prof. Kim's office was always open whenever I ran into a trouble spot or had a question about my research or writing. He has consistently driven me to push my limits, taught me so much, and steered me in the right direction. I am also gratefully indebted to him for his constant support and very valuable comments on this thesis.

I would also like to thank Niket Prakash and Dr. Jason B. Siegel of the College of Engineering at the University of Michigan in Ann Arbor for contributing to my work and providing me with experimental results and answers for any enquiry I had.

I would also like to thank Dr. Denise M. Rizzo of TARDEC for the project support.

Special thanks to Professor Taehyun Shim and Assistant Professor Stanley Baek for taking from their time to go through this thesis work and being part of the thesis defense committee.

I would also like to acknowledge Administrative Assistant and Graduate Secretary Sherry Boyd of the College of Engineering and Computer Science at the University of Michigan in Dearborn for her constant support and for always answering any doubts and questions I had throughout my degree program. 


\section{Table of Contents}

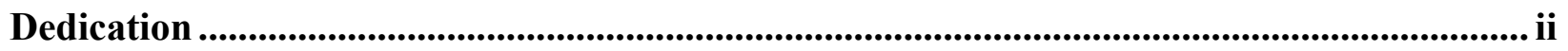

Acknowledgements .............................................................................................................................ii

List of Figures............................................................................................................................ $\mathrm{v}$

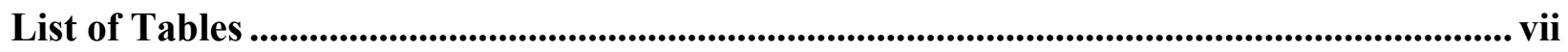

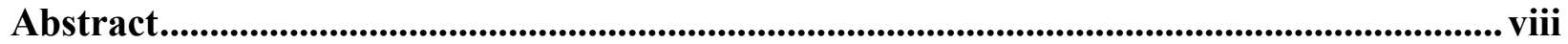

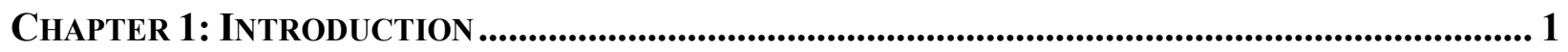

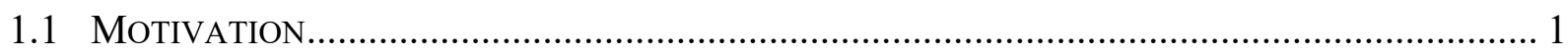

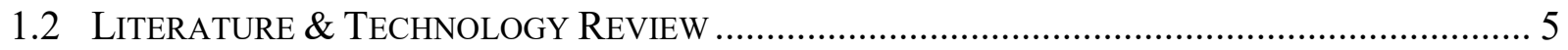

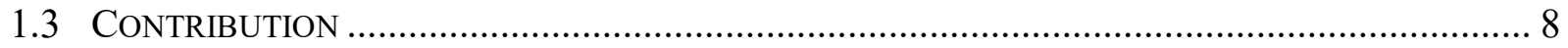

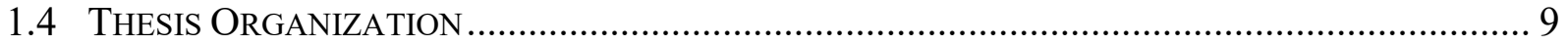

ChAPTER 2: MiNIMUM ENERGY CONSUMPTION PROBLEM .......................................................... 11

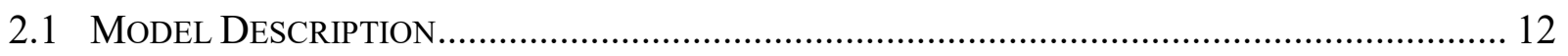

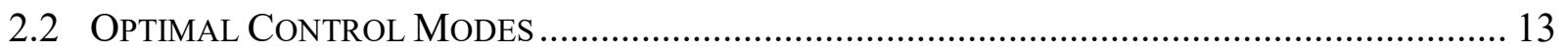

2.3 CONSTRAINTS ON PROPULSION AND BRAKING.............................................................. 17

Chapter 3: Dynamic Programming IMPlementation \& CaSe STUdies ............................ 20

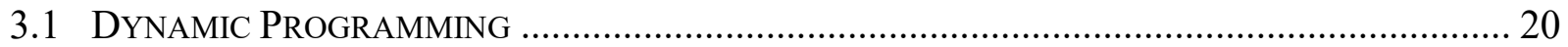

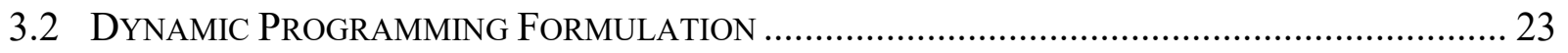

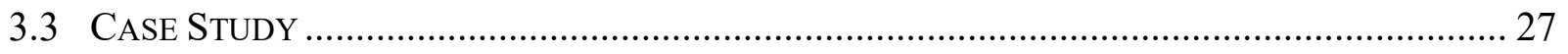

Chapter 4: Influence of Powertrain Design on Performance and Control Modes

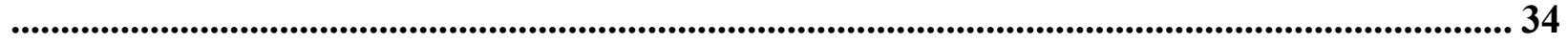

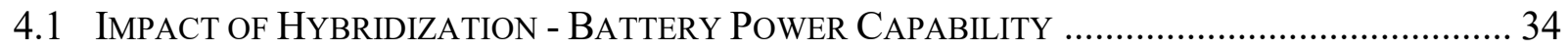

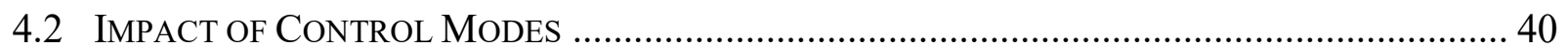

4.3 IMPACT OF EFFICIENCIES ON CONTROL MODES ............................................................ 41

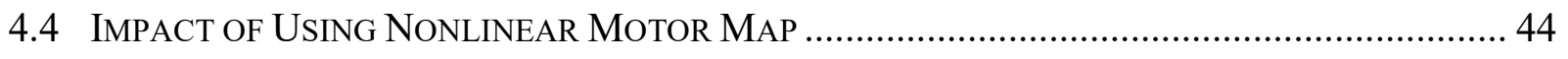

CHAPTER 5: CONCLUSION AND FUTURE WORK ........................................................................... 53

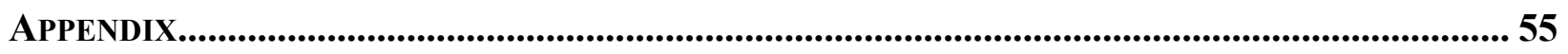

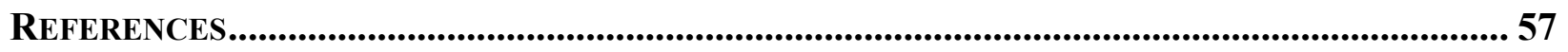




\section{List of Figures}

Figure 1 - Factors that can Reduce Oil Use in Different States [2] .......................................... 2

Figure 2 - Schematic of Hybrid Vehicle Major Components ………............................................. 3

Figure 3 - Eco-Driving Technique with Highlight on Thesis Focus ............................................... 5

Figure 4 - Schematic of Maximum Force Curve as a Function of Velocity................................... 18

Figure 5 - Sample Model of Considered Lightweight Military Robot [64].................................. 27

Figure 6 - Baseline Military Drive Cycle: Convoy Cycle ....................................................... 29

Figure 7 - Tradeoffs Between Energy Consumption and Trip Time for Convoy Cycle ............. 30

Figure 8 - Comparison of Speed Profiles Over Convoy Cycle .................................................... 31

Figure 9 - Baseline Military Drive Cycle: Churchville B Cycle ………………………....... 31

Figure 10 - Tradeoffs Between Energy Consumption and Trip Time for Churchville B Cycle .. 32

Figure 11 - Comparison of Speed Profiles Over Churchville B Cycle ......................................... 33

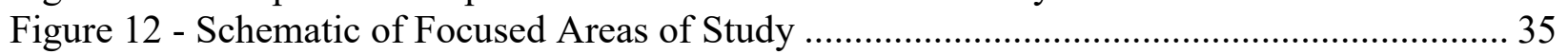

Figure 13 - Influence of Regen-Capability on the Energy Consumption and Driving Time:

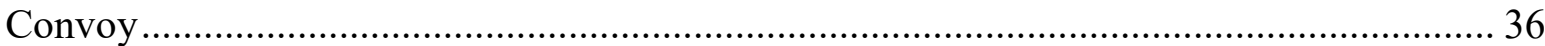

Figure 14 - Influence of Regen-Capability on the Energy Consumption and Driving Time:

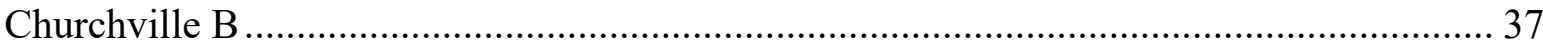

Figure 15 - Percentage Saving in Energy Consumption by Increasing Regen-Capability Over

Churchville B Cycle ..................................................................................................... 38

Figure 16 - Comparison of Operating Points for Different Regenerative Braking Capability Over

Churchville B Cycle for a Trip Time of 350 Seconds .......................................................... 39

Figure 17 - Histogram of Control Modes for Different Trip Times with Various Regenerative

Capability: $\quad 10 \mathrm{~kW}$ (Top); $15 \mathrm{~kW}$ (Middle); And $20 \mathrm{~kW}$ (Bottom) …………………... 41

Figure 18 - Impact of Efficiencies on Control Modes for Convoy Cycle ..................................... 43

Figure 19 - Impact of Efficiencies on Control Modes for Churchville B Cycle............................ 44

Figure 20 - Motor Efficiency Map..................................................................................... 45

Figure 21 - Sensitivity Comparison between Different DP Approaches for the Convoy Cycle .. 46

Figure 22 - Speed Profile and Efficiency Plot Using Constant and Nonlinear motor efficiency for

the Convoy Cycle

Figure 23 - Control Modes Comparison Between Nonlinear Efficiency Approach and Constant

Efficiencies for Convoy Cycle........................................................................................... 48

Figure 24 - Histogram Comparing Control Modes of Nonlinear Efficiency vs. Constant

Efficiencies Over Time for Convoy Cycle ....................................................................... 49

Figure 25 - Sensitivity Comparison between Different DP Approaches for the Churchville B

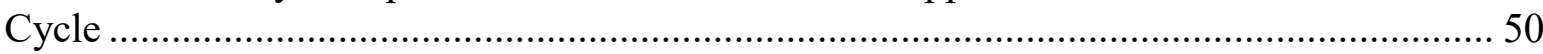

Figure 26 - Speed Profile Using Constant and Nonlinear motor map for the Churchville B Cycle

Figure 27 - Control Modes Comparison Between Nonlinear Efficiency Approach and Constant

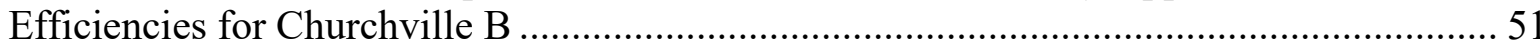


Figure 28 - Histogram Comparing Control Modes of Nonlinear Efficiency vs. Constant Efficiencies Over Time for Churchville B Cycle ……………............................................ 52 


\section{List of Tables}

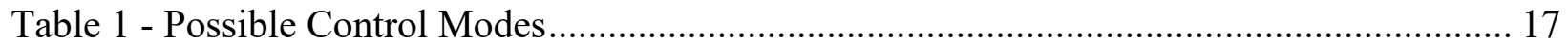

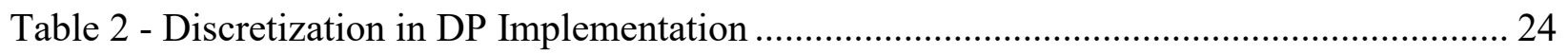

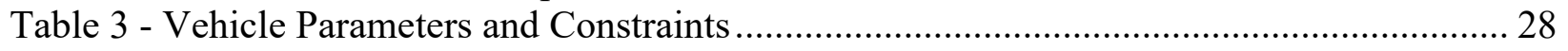




\begin{abstract}
This work presents a study of the energy-efficient operation of all-electric vehicles leveraging route information, such as road grade, to adjust the velocity trajectory. Minimization of energy consumption is one of the main targets of research for both passenger vehicles in terms of economic benefit, and army vehicles in terms of mission success and decision making. The optimization of a speed profile is one of the tools used to achieve energy minimization and it can also help in the useful utilization of autonomy in vehicles. The optimization of speed profile is typically addressed as an Optimal Control Problem (OCP). The obstacle that disrupts the implementation of optimization is the heavy computational load that results from the number of state variables, control inputs, and discretization, i.e., the curse of dimensionality. In this work, Pontryagin's Maximum Principle (PMP) is applied to derive necessary conditions and to determine the possible discrete operating modes. The analysis shows that only five modes are required to achieve minimum energy consumption; full propulsion, cruising, coasting, full regeneration, and full braking. Then, the problem is reformulated and solved in the distance domain using Dynamic Programming to find the optimal speed profiles. Various simulation results are shown for a lightweight autonomous military vehicle. Army Programs use various drive cycles including time, speed, and grade, for testing and validating new vehicle systems and models. Among those cycles, two different drive conditions are studied: relatively flat, Convoy, and hilly terrain, Churchville B. For the Convoy cycle, the optimal speed cycle uses $21 \%$ less energy for the same trip duration or reduces the time by $14 \%$ with the same energy consumption while for the Churchville B cycle, it uses $24 \%$ less energy or provides $24 \%$ reduction in time. Furthermore, the sensitivity of energy Viii
\end{abstract}


consumption to regenerative-braking power limits and trip time is investigated. These studies provide important information that can be used in designing component size and scheduling operation to achieve the desired vehicle range. Lastly, the work provides parametric studies about the influence of the efficiency of an electric motor on performance including energy consumption and control modes. 


\section{Chapter 1: Introduction}

\subsection{Motivation}

The automotive industry is in constant challenge of developing sustainable solutions to the challenges of energy exhaustion and environment pollution. There is a continuous need to effectively improve energy efficiency and reduce energy consumption. Vehicle fuel consumption is not only related to the performance of the vehicle itself but also related to the drivers' driving behavior [1]. Additionally, increasing the efficiency of hybrid-electric vehicles helps decrease the dependence on imported oil and aids in the move towards cleaner energy. The technology available in the automotive industry today holds substantial potential that can be manifested through optimization. Optimization can be reflected in terms of energy consumption, sizing of vehicle components, and utilizing autonomous vehicles. Improving the fuel efficiency of vehicles is a vital step that can cut the oil consumption in half. In fact, doubling the fuel efficiency of new cars, trucks, and commercial vehicles in the U.S alone would, by 2035, save over 5 million barrels of oil per day [2]. Figure 1 displays a sample of factors that can help reduce oil use in some of the states. 

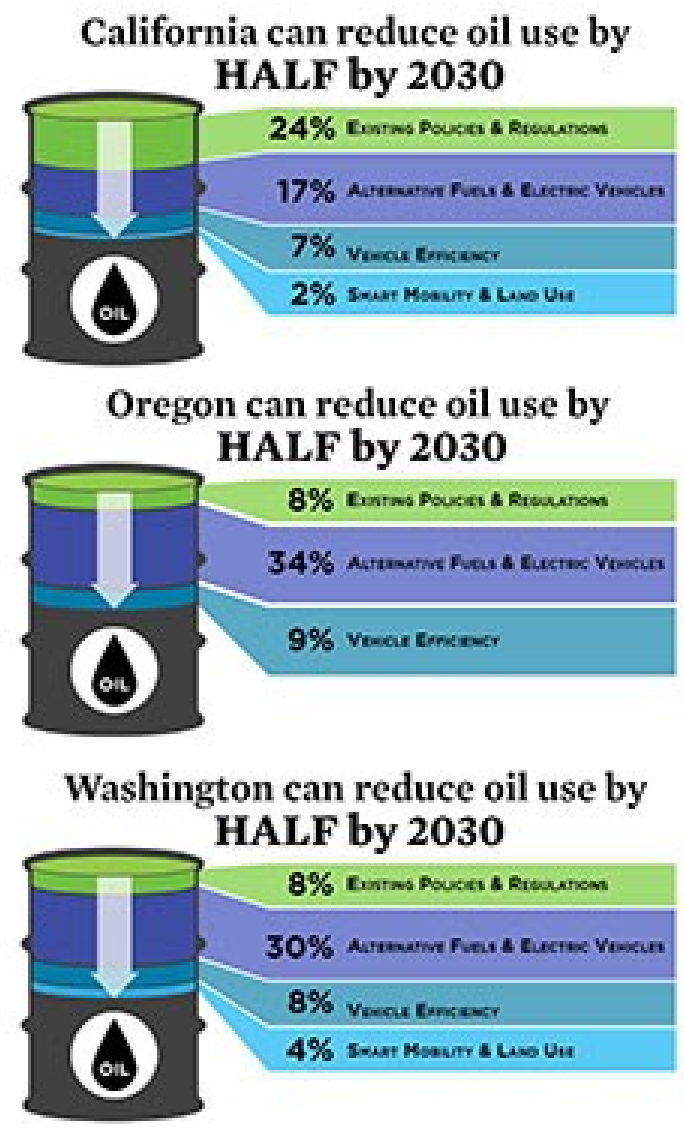

Figure 1 - Factors that can Reduce Oil Use in Different States [2]

Minimization of energy consumption is one of the main goals of this research and it is critical for both passenger vehicles in terms of economic benefit, and army vehicles in terms of mission success and decision making. When it comes to military vehicles, they are required to: (1) be more fuel efficient, (2) provide robust performance, and (3) have the ability to support a variety of electrical loads. Hybrid drivetrains have been receiving attention in all branches of the military (ships, airplanes, combat vehicles etc.) for their potential strategic benefits. A great demand has been set to evolve the drivetrain configurations and develop better control strategies. Optimizing fuel consumption for these ground vehicles can be of great benefit on the field. Minimizing energy consumption is still a huge challenge for ground vehicles. Powertrain technology has been in continuous improvement; nonetheless, drivers have not adapted their driving style to improve 
efficiency. Eco-driving or energy-efficient driving is the term given to the idea of determining the speed trajectory that minimizes the vehicle energy consumption under final time and distance constraints. Utilizing such data can provide the necessary information to operate the vehicle in an energy-efficient manner. Vehicle electrification is one of the promising technologies that improves fuel economy, tailpipe emissions, and sustainability. The number of electrified vehicles including hybrid electric vehicles (HEVs), Plug-in hybrid electric vehicles (PHEVs) and Electric Vehicles (EVs) is expected to keep increasing [3]. When it comes to fuel-cell hybrid vehicles, there is a $34 \%$ increase in sales in the 1st quarter of 2018 compared to the 1st quarter of 2017 [4]. Finding the most efficient component size in this kind of vehicles is a real challenge that withholds many variables. Figure 2 displays a schematic of the major components of a hybrid vehicle.

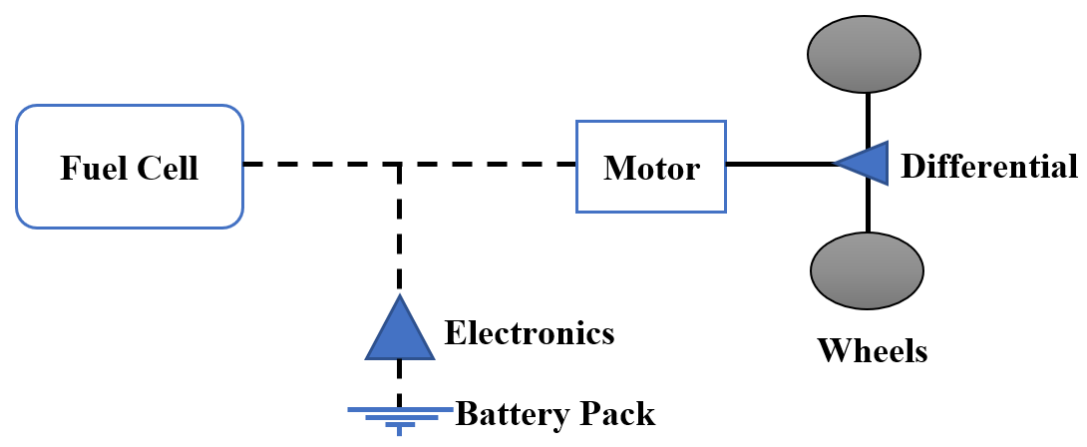

Figure 2 - Schematic of Hybrid Vehicle Major Components

The technologies and advancements available in the automotive field make the search not only to find the next technological breakthrough, but also to make the most out of the what is presently available. The vehicles available today have the sensors, lidars, software, and capabilities to predict the estimated time and distance of a trip based on the drivers' chosen destination and real-time traffic data. Utilizing such data can provide the necessary information to operate the vehicle in an energy-efficient manner. The aim of eco-driving is to procure the most efficient solution that involves both the vehicle speed and the vehicle surroundings, including road environment and traffic flow, through the appropriate operation controlled by a driver or an 
autonomous vehicle. Eco-driving is an effective tool that pertains vehicle parameters, such as vehicle mass and resistive forces, to minimize fuel use [1]. Research on enhancing the energy efficiency of vehicles in general and of autonomous driving systems in specific have been studied for the past four decades $[5,6]$. The work has involved addressing the challenges that lie in driver's behavior, decision making, path planning, and trajectory generation $[1,7,8,9]$. Traditionally, speed profile optimization has been extensively studied in application to railway vehicles because of their operating environment: a given route, known road grade, and speed limits [10, 11, 12]. Hybrid electric vehicles (HEVs) are superior to conventional vehicles in terms of the benefit of combining several propulsion components as a way of producing a more efficient vehicle in terms of saving energy and reducing pollution. A typical hybrid vehicle design takes a conventional vehicle drivetrain and combines it with components commonly found in an electric vehicle. In a fuel cell hybrid, a different approach is taken to harness the electrochemical energy produced by the fuel cell. The process involves hydrogen being converted into electrical energy that drives the wheels of an electric vehicle. Recent developments has shown that fuel cell hybrid vehicles can provide high efficiency, high vehicle fuel economy, very low emissions, and a mileage of more than 300 miles. The U.S. Department of Energy is leading government and industry efforts to make hydrogen-powered vehicles an affordable, environmentally friendly, and safe transportation option $[13,14]$. With these efforts, it is important for the fuel cell hybrid vehicles to be optimized to their best of their potential. Additionally, the increasing costs for fuel and transportation logistics creates a growing interest in improving fuel efficiency. The employment of hybrid drivetrain architecture and effective power management of multiple power sources proves to be an effective solution to address the problem [15]. However, efficient solutions need to meet the rapidly increasing needs of transportation vehicles in general, and military vehicles 
in specific. Military vehicles are in greater necessity to be more fuel efficient, provide robust performance, and work with variety of power sources. Hybrid drivetrains have been receiving greater attention for their potential strategic benefits and multiple power sources. Solving a power management problem that optimizes fuel consumption for these ground vehicles can be of great benefit on the field $[15,16]$.

\subsection{Literature \& Technology Review}

When it comes to eco-driving, there are different study fields that researchers focus on. Figure 3 shows a schematic of some of the major eco-driving branches, with the study field addressed in this thesis highlighted.

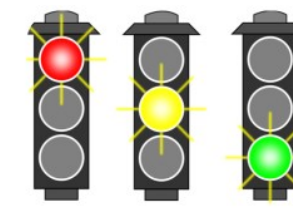

Anticipation of Traffic Signal

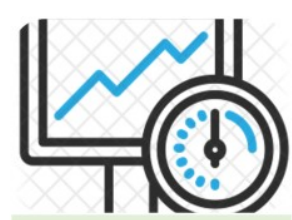

Speed Optimization

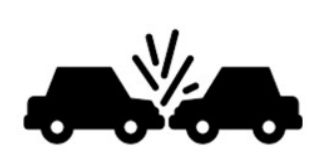

Safety and Crash Avoidance

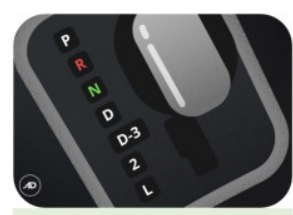

Control Driving Modes

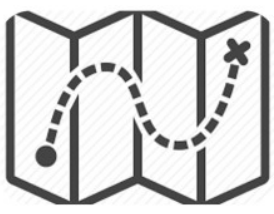

Path Planning

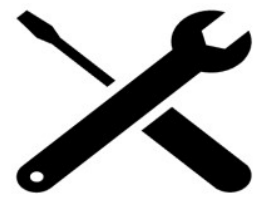

Powertrain Improvement

Figure 3 - Eco-Driving Technique with Highlight on Thesis Focus

Vehicle trajectory optimization and control have been extensively studied in a broader domain, including surface vehicles, aircraft and Unmanned Aerial Vehicles (UAV) [17-19]. The first work on optimal control and optimal speed profiles was done by Schwarzkopf and Leipnik [5]. In their work, Pontryagin's Maximum Principle (PMP) was used to develop a mathematically optimal performance subject to the driver's choice of a trip time limit that could reduce fuel consumption. The work also provided detailed solutions that reflected that constant speed is optimal for level 
roads and highways. This work set a base for the study conducted in this thesis work as PMP is used but for the sake of finding the optimal control modes that operate the vehicle throughout the trip. Optimal speed profiles have been researched for different vehicles and transportations including off-road vehicles [20], and vehicles moving on fixed routes, such as trains [21], buses [22], or race cars [23], [24]. When the driving conditions are fixed or known prior to moving, optimal speed profiles can be calculated offline with an acceptable confidence and possibly implemented in an open-loop or state-feedback controller [25]. On the other hand, passenger vehicles on open roads experience a wide range of unknown driving conditions. Trip destination and route information, such as altitude, can be known in advance with the use of navigation system. Several studies [26-31] focus on applications of vehicles driven on highways, with high average speeds and varying road slopes. The speed profile can be adjusted to anticipate altitude variations depending on the route information. Hellström et al. [27] and [32] performed optimization of the velocity trajectory with respect to a criterion formulation that weighs trip time and fuel consumption. They also used a predictive control scheme based off dynamic programming algorithm to constantly feed the conventional cruise controller with new set points that reflect optimal results. Moreover, Kamal et al. [9] provided a nonlinear model predictive control method with a fast optimization algorithm that derives the vehicle control inputs based on road gradient conditions obtained from digital road maps. Additionally, Li et al. [33] recently developed a Driver Advisory Systems (DAS) that aims to provide efficient driving instructions to the driver with optimized trajectory. Their work results in a trajectory optimization method that achieves energy efficiency and punctuality for DAS through the use of offline and online optimization together. Moreover, another development in the field of optimization and optimal control has been developed by Ding and Jin [1] that provided optimal speed profiles that can minimize fuel 
consumption for a vehicle travelling on a curve. The work conducted in this thesis uses offline computation of speed profile to minimize energy consumption on a flat and hilly path. The problem solved in this work has been addressed as an Optimal Control Problem (OCP) with related work done by researchers [33-52]. The solutions of which can provide a vehicle speed profile that decreases the amount of energy consumed over the trip.

In solving the trajectory optimization problem, numerical approaches such as Genetic Algorithm [33,42,44], Ant Colony Algorithm [44], Sequential Quadratic Programming [41], Nonlinear Programming [51] and Dynamic Programming (DP) [35-39] have been used for nonlinear characteristics, such as varying speed limits, resistive forces. Among these, DP has been widely and extensively used because it can find a global optimum even for nonlinear systems with nonlinear constraints. In [50], authors applied DP to determine optimal torque distribution between front and rear in-wheel motors with preview terrain information. However, these methods typically require substantial computation time due to discretization of states and control inputs. In [51,52], the authors propose approaches to improve computational efficiency through approximated vehicle operation and problem reformulation, respectively. Pontryagin's Maximum Principle (PMP) can be used to analyze the system and to decrease the computational cost by reducing the number of possible control actions to the set which achieves the optimal solution. Different cases have been studied for different kinds of vehicles in order to increase the efficiency. For instance, authors in $[48,49]$ approached the problem to minimize fuel consumption and engine emissions for conventional vehicles. Sciarretta et al. [23] consider the hybrid vehicles in their studies as they work on finding the fuel optimal trajectory for a fuel cell powered vehicle. They find an optimal velocity profile for a trip but with fixed average speed by applying numerical optimization methods. DP optimization was also applied to optimize fuel consumption with considerations of 
the terrain's grade angle by Tsao [53]. Nonetheless these works did not involve the use of any methods to decrease computational load. Lastly, standard results for the minimization of energy consumption at wheels for vehicles such as in the work done by Sciarretta et al. [25] consist of only four modes: full propulsion, cruising, coasting, and braking.

\subsection{Contribution}

The optimal control modes of all-electric vehicles may not necessarily be the same as those of conventional vehicles. This work extends the basic idea of applying PMP to all-electric vehicles and synthesizes the results of the PMP analysis into DP formulation. When applying PMP, three control inputs are deliberately introduced: motoring, regenerating and friction braking, unlike approaches in [36,38]. For the given vehicle model, namely, point-mass longitudinal dynamics with invariant motor efficiency, five modes are required to achieve energy-efficient operation: full propulsion, cruising, coasting, full regeneration, and full regeneration with conventional braking. Based on this result, the minimum energy consumption problem is reformulated and solved by DP for the case of a lightweight military vehicle. In particular, two different driving environments including non-hilly and rugged roads are studied. The simulation results demonstrate the effectiveness of eco-driving in regards to energy consumption and driving time, which could be useful in planning mission trips. Furthermore, this work investigates the sensitivity of performance such as net energy consumption and journey time to regenerative braking power capability, typically not considered in speed optimization problems. As regenerative braking capability changes, for example, because of the size of batteries or thermal management system malfunction, the optimal trajectory may change. Thus, the optimal trajectory planning accounting for regenerative braking power would provide beneficial information in both design and control aspects. Moreover, efficient use of control modes has a significant impact on the overall energy 
consumption and vehicle performance. The importance of the operational environment also plays an important role in the mode changes that occur. Additionally, the constraints of deceleration, acceleration, speed, and distance limit, also play an essential role in the selection of control modes. The impact of battery sizing and regenerative power impact on the change of control modes is reflected in this paper. Additionally, the work provides parametric studies that involve the inclusion of global optimal solution based off nonlinear motor map. The sensitivity of results is compared to constant efficiency and suboptimal solution from nonlinear efficiency map. The results add value in the understanding of the accuracy of results based off assumptions made. The work in this thesis is contributed with the works done in the following publications:

1. H. Abbas, Y. Kim, J.B. Siegel, D.M. Rizzo, "Optimization of Energy-Efficient Speed Profile for Electrified Vehicles", Dynamic Systems and Control Conference, Atlanta, 2018.

2. H. Abbas, Y. Kim, J.B. Siegel, D.M. Rizzo. 2018. Synthesis of Pontryagin's Maximum Principle Analysis for Speed Profile Optimization of Electrified Vehicles, Journal of Dynamic Systems, Measurement, and Control, to appear.

\subsection{Thesis Organization}

This thesis is organized as follows: Chapter II describes a detailed modeling of a ground vehicle and analysis of the optimal control problem. Synthesis of Pontryagin's Maximum Principle for speed profile optimization is analyzed. The reformulation and implementation in Dynamic Programming is further described in Chapter III. Case studies are presented in application of a light weight military vehicle, and trajectory optimization results obtained by DP are discussed. In Chapter IV, the impact of battery sizing and regenerative power impact on the change of control modes is investigated. The last section of the chapter focuses on parametric studies that involve the inclusion of global optimal solution based off nonlinear motor map. The sensitivity of results 
is compared to constant efficiency and suboptimal solution from nonlinear efficiency map. The results could provide a deeper understanding of the accuracy of results that are obtained with the use of some assumptions. The chapter also includes analysis of the sensitivity of results comparing suboptimal solutions and global optimal solutions. Finally, Chapter V provides a conclusion and direction for future work. 


\section{Chapter 2: Minimum Energy Consumption Problem}

In this section, the minimum energy consumption problem is presented and analytically solved by applying Pontryagin's Maximum Principle (PMP). PMP is a powerful computational and analytical tool used to solve optimal control problems. PMP provides a set of necessary conditions that an optimal control must satisfy while maximizing (or minimizing) the Hamiltonian function. Despite the fact that PMP generally yields necessary conditions, it is still useful and effective (1) to find a small subset of control actions and (2) to numerically compute optimal control trajectory significantly fast compared to DP. For a certain class of optimal control problems, such as power management of hybrid electric vehicles, solutions obtained from PMP and DP are hardly different, which makes PMP widely used in automotive applications [49,54,55]. For simplicity, the following assumptions are made: (1) the efficiency of the system is invariant, that is, motoring and generating efficiency of the electric motor is fixed; (2) the vehicle moves forward only; (3) road grade and velocity limit are distance-dependent and known a priori. It is noted that although the efficiency of an electric motor is generally nonlinear [50], the efficiency is relatively constant above certain force or torque levels covering nominal operating range, which makes the first assumption reasonable. Chapter IV of this thesis displays the results of the analysis using nonlinear efficiency motor map. 


\subsection{Model Description}

The motion of a ground vehicle considered in this study is described by the following equations:

$$
\begin{aligned}
& \frac{d s}{d t}=v, \\
& M \frac{d v}{d t}=F_{p}+F_{b}-A-B v-C v^{2}-M g \sin \theta,
\end{aligned}
$$

Where $s$ and $v$ represent distance and velocity respectively while $M$ is vehicle mass and $t$ is time. The variables $A, B$, and $C$ are coefficients used to determine resistance forces by rolling and aerodynamic drag, and the grade angle $\theta$ is a function of distance, i.e. $\theta=\theta(s)$. The variables $F_{p}$ and $F_{b}$ denote control inputs regarding propulsion and braking, respectively. Hereafter, $\bar{*}$ and $*$ are used to refer to the maximum and minimum values of parameter $*$.

For the purpose of OCP analysis, Eq. (1b) is rewritten as:

$$
M \frac{d v}{d t}=F_{m}+F_{g}+F_{f b}-A-B x_{2}-C x_{2}^{2}-M g \sin \theta
$$

Where $F_{m}, F_{g}$, and $F_{f b}$ represent motoring, generating, and friction braking forces, respectively. This choice is deliberate to clearly distinguish operational modes of the vehicle in the OCP analysis discussed later. By defining states and controls such that:

$$
\begin{aligned}
& x=[s, v]^{T}, \\
& u=\left[u_{1}, u_{2}, u_{3}\right]^{T}=\left[\frac{F_{m}}{M}, \frac{F_{g}}{M}, \frac{F_{f b}}{M}\right]^{T},
\end{aligned}
$$

the equations of motion (1) and (2) can be expressed as following:

$$
{ }_{2}+u_{3}-\alpha \cos \theta\left(x_{1}\right)-\beta x_{2}-\gamma x_{2}^{2}-g \sin \theta\left(x_{1}\right)
$$


Where $\alpha=\frac{A}{M}, \beta=\frac{B}{M}, \gamma=\frac{C}{M}$. The coefficients $A, B$, and $C$ are used to compute the resisting forces; rolling and aerodynamics forces, with the resisting forces being speed-dependent, whilst the grade force depends on the angle, $\theta$, such that:

$$
\begin{aligned}
& f_{r}=A+B x_{2}+C x_{2}{ }^{2} \\
& f_{g}=M g \sin \theta
\end{aligned}
$$

Control inputs are bounded by their limits such that $0 \leq u_{1} \leq u_{1}\left(x_{2}\right), u_{2}\left(x_{2}\right) \leq u_{2} \leq 0$, and $u_{3}\left(x_{2}\right) \leq u_{3} \leq 0$. It is noted that the force limits of an electric machine and a friction brake are typically functions of speed. Initial and terminal conditions of the states are:

$$
\begin{array}{ll}
x_{1}(0)=0, & x_{2}(0)=0, \\
x_{1}\left(t_{f}\right)=S_{f}, & x_{2}\left(t_{f}\right)=0
\end{array}
$$

Where $t_{f}$ and $S_{f}$ are operational time and distance traveled, and these are bounded, meaning that the vehicle stops at time $t_{f}$ after traveling a given distance, $S_{f}$. The vehicle is assumed to move forward only, i.e., $x_{2}(t)>0, t \in\left(t_{0}, t_{f}\right)$.

\subsection{Optimal Control Modes}

The primary focus of this study is to optimize a speed profile which minimizes the energy consumption while traversing a given distance. Therefore, the cost function to be minimized is the normalized total energy consumption fora given mission and defined by:

$$
J=\int_{0}^{t_{f}} \frac{P_{e}^{\text {elec }}}{M} d t=\int_{0}^{t_{f}}\left(x_{2} u_{1} \eta_{1}+x_{2} u_{2} \eta_{2}\right) d t
$$

Where $P_{e}^{e l e c}$ is the electrical power by the motor; and $\eta_{1}$ and $\eta_{2}$ denote motoring and generating efficiency of the electrical machine, respectively. The Hamiltonian is defined as: 


$$
\begin{aligned}
H=\frac{x_{2} u_{1}}{\eta_{1}} & +\eta_{2} x_{2} u_{2}+p_{1}\left(x_{2}\right)+p_{2}\left(u_{1}+u_{2}+u_{3}\right. \\
& \left.-\alpha \cos \theta\left(x_{1}\right)-\beta x_{2}-\gamma x_{2}^{2}-g \sin \theta\left(x_{1}\right)\right)
\end{aligned}
$$

The corresponding ad-joint equations are written as:

$$
\begin{array}{ll}
i & \frac{g(x)}{\partial x_{1}} \\
i \quad i_{1}-\eta_{2} u_{2}-p_{1}\left(x_{2}\right)+p_{2} \frac{\partial g(x)}{\partial x_{2}}
\end{array}
$$

Where $\theta^{\prime}=\frac{\partial \theta\left(x_{1}\right)}{\partial x_{1}}$.

The Hamiltonian function is further simplified by factoring out the control input $u$, in order to determine the driving modes. Then equation 6 becomes:

$$
\begin{aligned}
H= & \left(\frac{x_{2}}{\eta_{1}}+p_{2}\right) u_{1}+\left(x_{2} \eta_{2}+p_{2}\right) u_{2}+p_{2} u_{3}+p_{1} x_{2} \\
& -\alpha p_{2} \cos \theta\left(x_{1}\right)-\beta p_{2} x_{2}-\gamma p_{2} x_{2}^{2}-p_{2} g \sin \theta\left(x_{1}\right)
\end{aligned}
$$

Based on the values of the switching functions, $\frac{x_{2}}{\eta_{1}}+p_{2}, x_{2} \eta_{2}+p_{2}$, and $p_{2}$, the control inputs to minimize $H$, and the fact that each have three options of being greater than, less than, or equal to $0, \underline{27}$ different modes are found possible. It should be noted that not all of the modes are feasible due to the assumptions made. In other words, infeasible mode scan be first ruled out by investigating the assumptions. First, efficiency of the electric motor must be greater than 0 and less than 1; i.e., $0<\eta_{i}<1, i \in\{1,2\}$. Second, the electric vehicle moves forward only, i.e., $x_{2}>0$. From these two conditions, it is found that $\frac{x_{2}}{\eta_{1}}>\eta_{2} x_{2}$. For instance, when $p_{2} \geq 0$, the conditions $\frac{x_{2}}{\eta_{1}}+p_{2}<0$ and $x_{2} \eta_{2}+p_{2}<0$ are infeasible because they violate $x_{2}>0$. The conditions $\frac{x_{1}}{\eta_{1}}+$ $p_{2}<0$ and $x_{2} \eta_{2}+p_{2}=0$ cannot occur simultaneously since they violate $\frac{x_{2}}{\eta_{1}}>\eta_{2} x_{2}$. For the same 
reason, the conditions $x_{2} \eta_{2}+p_{2} \geq 0$ and $\frac{x_{2}}{\eta_{1}}+p_{2}<0$ cannot occur simultaneously as well. After the infeasible cases, found in Appendix A, are ruled out, the remaining seven cases that could result in feasible modes are obtained as follows:

$$
\begin{array}{llll}
\frac{x_{2}}{\eta_{1}}+p_{2}<0, \quad x_{2} \eta_{2}+p_{2}<0, \quad p_{2}<0 & : u=\left[u_{1}, 0,0\right]^{\prime} \\
\frac{x_{2}}{\eta_{1}}+p_{2}>0, \quad x_{2} \eta_{2}+p_{2}<0, \quad p_{2}<0 & : u=[0,0,0]^{\prime} \\
\frac{x_{2}}{\eta_{1}}+p_{2}>0, \quad x_{2} \eta_{2}+p_{2}>0, \quad p_{2}<0 & : u=[0, u 2,0]^{\prime} \\
\frac{x_{2}}{\eta_{1}}+p_{2}>0, \quad x_{2} \eta_{2}+p_{2}>0, \quad p_{2}>0 & : u=[0, u 2, u 3]^{\prime} \\
\frac{x_{2}}{\eta_{1}}+p_{2}=0, \quad x_{2} \eta_{2}+p_{2}<0, \quad p_{2}<0 & : u=[u n d e f, 0,0]^{\prime} \\
\frac{x_{2}}{\eta_{1}}+p_{2}>0, \quad x_{2} \eta_{2}+p_{2}=0, \quad p_{2}<0 & : u=[0, \text { undef }, 0]^{\prime} \\
\frac{x_{2}}{\eta_{1}}+p_{2}>0, \quad x_{2} \eta_{2}+p_{2}>0, \quad p_{2}=0 & : u=[0, \text { u2,undef }]^{\prime}
\end{array}
$$

For the last three cases, $u_{1}, u_{2}$, and $u_{3}$ are undefined because the optimal control mode is not uniquely determined from the given condition. The existence of the optimal control mode can be analyzed by checking the necessary condition, known as Kelley's condition [56]. For a single control problem, the control $u$ for a singular-arc can be determined by differentiating $\xi=\frac{\partial H}{\partial u}$ with respect to time $t$ until $u$ appears explicitly, and then Kelley's condition must be satisfied. That is:

$$
\begin{array}{r}
\frac{\partial^{i} \xi}{\partial t_{i}}=0, \quad i=0,1, \ldots . ., 2 l-1, \\
\frac{\partial^{2 l} \xi}{\partial t^{2 l}}=h_{1}(x, p)+h_{2}(x, p) u, \\
(-1)^{l+1} \frac{\partial}{\partial u}\left(\frac{\partial^{2 l} \xi}{\partial t^{2 l}}\right) \leq 0
\end{array}
$$


Where $l$ is an integer variable. In the case $(9 \mathrm{e}), u_{1}$ is undefined. Consider $\xi_{1}=\frac{\partial H}{\partial u_{1}}=\frac{x_{2}}{\eta_{1}}+p_{2}=0$. Its first- and second-time derivatives are given by:

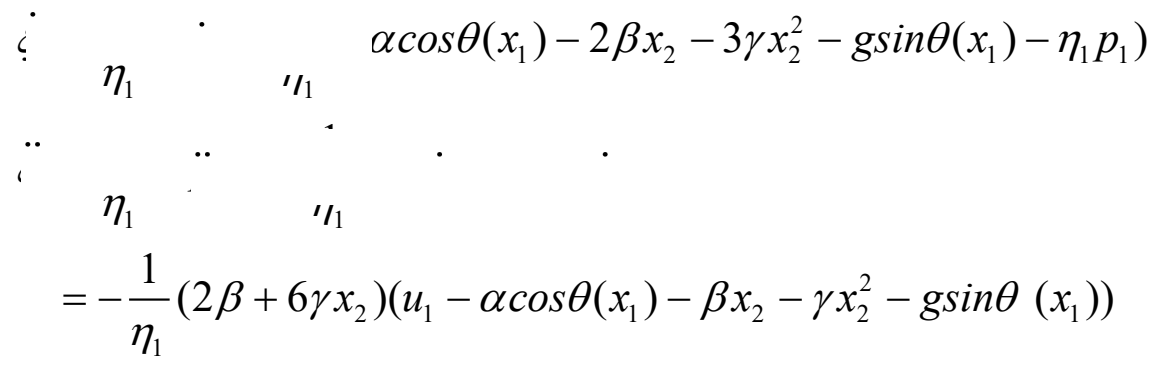

Since $2 \beta+6 \gamma x_{2} \neq 0$, the candidate solution is obtained as

$$
u_{1}=\alpha \cos \theta\left(x_{1}\right)+\beta x_{2}+\gamma x_{2}^{2}+g \sin \theta\left(x_{1}\right)
$$

Moreover, this solution satisfies the Kelley's condition:

$$
(-1)^{2} \frac{\partial}{\partial u_{1}} \frac{d^{2}}{d t^{2}} \xi_{1}=\frac{-2 \beta-6 \gamma x_{2}}{\eta_{1}} \leq 0
$$

Similarly, in the case (9f), the solution can be found to be:

$$
u_{2}=\alpha \cos \theta\left(x_{1}\right)+\beta x_{2}+\gamma x_{2}^{2}+g \sin \theta\left(x_{1}\right)
$$

The cases (9e) and (9f) are both cruising mode as the same amount of input is applied to the system in order to keep it at constant speed; (9e) applies the input in propulsion while (9f) applies it in braking.

For case $(9 \mathrm{~g}), u_{3}$ is undefined. Consider $\xi_{3}=\frac{\partial H}{\partial u_{3}}=p_{2}=0$. Then, its first- and second-time derivatives are ex-pressed as following:

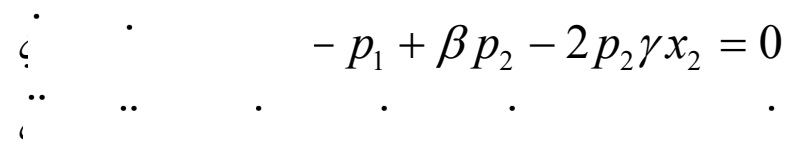


Since $p_{2}=0, \dot{p}_{1}=0$ from Eq. (7a), the solution would never reach a situation in which $u_{3}$ (friction braking) would stand alone and have a distinct solution; therefore, this mode is also infeasible. Finally, the list of the remaining excluded control modes is summarized in Table 1.

Note that the number of control modes is five and that two different braking modes are used in an electrified vehicle unlike a conventional vehicle without regenerative braking capability.

Table 1 - Possible Control Modes

\begin{tabular}{|c|c|}
\hline Control $u=\left[u_{1}, u_{2}, u_{3}\right]$ & $\underline{\text { Description }}$ \\
\hline$\left[\overline{u_{1}}, 0,0\right]$ & Full Propulsion \\
\hline$[0,0,0]$ & Coasting \\
\hline$\left[0, \underline{u_{2}}, 0\right]$ & Full Regenerative Braking \\
\hline$\left[0, \underline{u_{2}}, \underline{u_{3}}\right]$ & Full Braking \\
\hline$\left[\alpha \cos \theta\left(x_{1}\right)+\beta x_{2}+\gamma x_{2}^{2}+g \sin \theta\left(x_{1}\right), 0,0\right]$ & Cruising \\
\hline$\left[0, \alpha \cos \theta\left(x_{1}\right)+\beta x_{2}+\gamma x_{2}^{2}+g \sin \theta\left(x_{1}\right), 0\right]$ & Cruising \\
\hline
\end{tabular}

\subsection{Constraints on Propulsion and Braking}

Propulsion and regenerative-braking of the electrified vehicle are limited by the maximum power from electric components such as electric motor(s), a fuel-cell and a battery. As a result, the influence of these limits or constraints on vehicle operation need to be clearly understood to properly formulate the control problem. In general, electric motors used in transportation system are characterized by the maximum torque curve, a function of their operating velocity as schematized in Figure 4. 


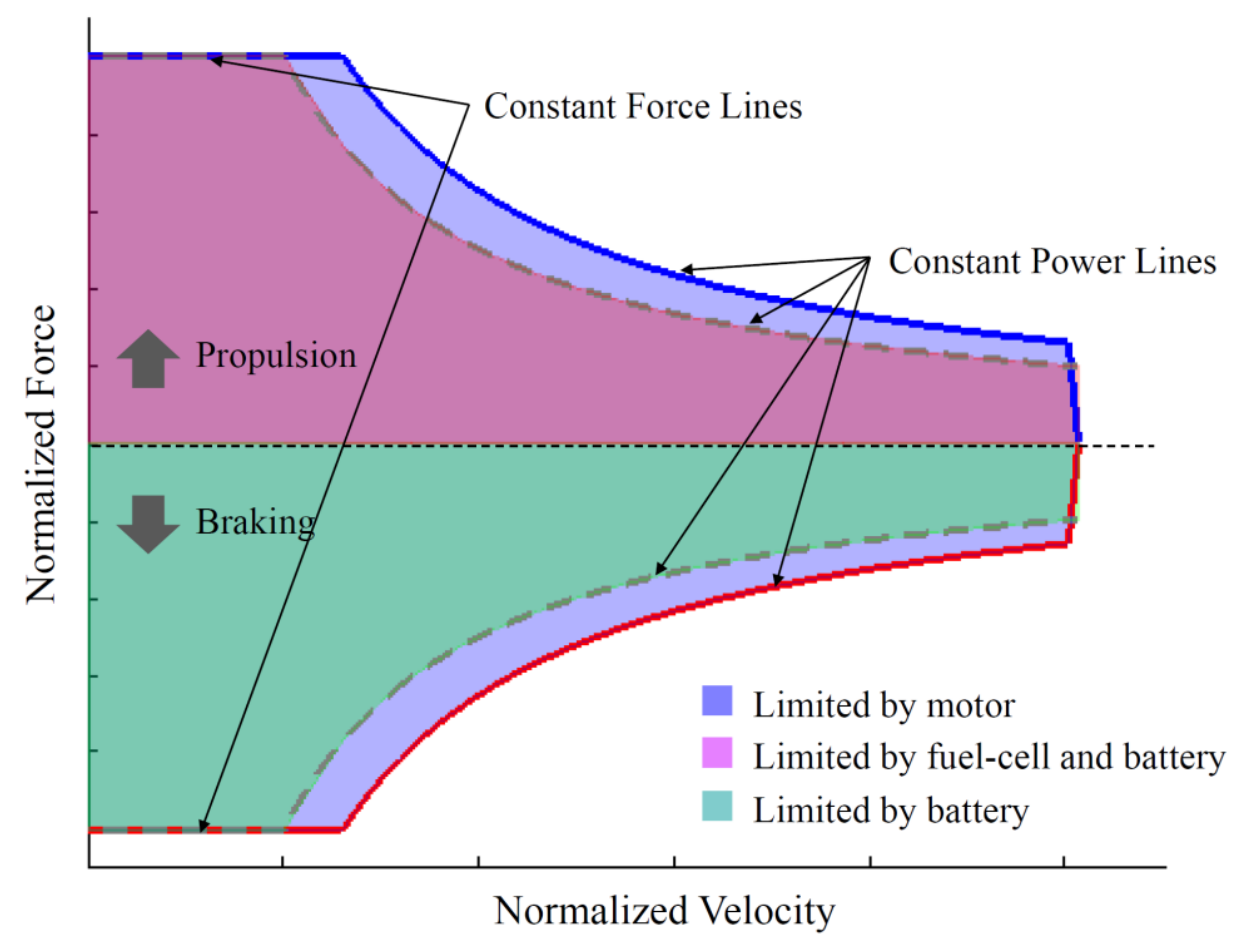

Figure 4 - Schematic of Maximum Force Curve as a Function of Velocity

The $v-f$ relationship can be obtained from the motor torque, $\tau$, the tire radius, $r$, and the final drive ratio $i_{a}$ :

$$
\begin{aligned}
& \bar{f}=\frac{\bar{\tau}}{r} i_{a} \\
& \underline{f}=\frac{\underline{\tau}}{r} i_{a}
\end{aligned}
$$

The force limit sets the reference value for the maximum propulsion and maximum regenerative braking that the vehicle is capable of. Moreover, the velocity of the vehicle is restricted by the limit of the motor's rotational speed, $\omega$, from the following relation:

$$
\bar{v}=\frac{\bar{\omega} r}{i_{a}}
$$

Therefore, the optimal trajectories of the vehicle should be found in consideration of the motor characteristics as given by: 


$$
\begin{aligned}
& 0 \leq u_{1} \leq \eta_{1} \varphi_{1}\left(x_{2}\right) \\
& \frac{\varphi_{2}\left(x_{2}\right)}{\eta_{2}} \leq u_{2} \leq 0
\end{aligned}
$$

Where $\varphi_{1}$ and $\varphi_{2}$ are velocity-dependent upper and lower limits of the force. However, the motor power is constrained by power/energy sources. For instance, when fuel-cells and batteries are used, motoring power is limited by the maximum power from both fuel-cells and batteries whereas charging power is limited by batteries only; in this case, the shape of force limits is no longer symmetric. The functions $\varphi_{1}$ and $\varphi_{2}$ are influenced by the size of powertrain components, i.e.,

$$
\begin{gathered}
\varphi_{1}=\min \frac{\left\{\frac{P_{b}+P_{f c}}{x_{2}}, \frac{P_{m}}{x_{2}}, f_{e}^{\bar{a}}\right\}}{M}, \\
\varphi_{2}=\max \frac{\left\{\frac{P_{b}}{x_{2}}, \frac{P_{m}}{x_{2}}, f_{e}^{a}\right\}}{M},
\end{gathered}
$$

with

$$
\begin{aligned}
& f_{e}^{\bar{a}}=\frac{\bar{a}-\alpha \cos \theta\left(x_{1}\right)-\beta x_{2}-\gamma x_{2}^{2}-g \sin \theta\left(x_{1}\right)}{\eta_{1}}, \\
& f_{e}^{a}=\eta_{2}\left(\underline{a}-\alpha \cos \theta\left(x_{1}\right)-\beta x_{2}-\gamma x_{2}^{2}-g \sin \theta\left(x_{1}\right)\right),
\end{aligned}
$$

Where $P_{b}, P_{f c}$ and $P_{m}$ denote battery power, fuel-cell power, and motor power, respectively. Note that the switching functions are the same as those described in Section 2.2; therefore, $u_{1}$ and $u_{2}$ become $\varphi_{1}\left(x_{2}\right)$ and $\varphi_{2}\left(x_{2}\right)$, respectively. The influence of propulsion and braking power on the optimal trajectory will be presented and discussed in Chapter IV. 


\section{Chapter 3: Dynamic Programming Implementation \& Case Studies}

The formulation of an optimal control problem is complicated and has implications for the obtained solution. By solving the same problem in different ways, we can determine more straightforward and less computationally intensive methods. Specific factors impact the optimization of energy consumption in an electric vehicle. These factors include the constraints on the speed, acceleration, time, distance, and braking force. Additionally, the problem can be formulated in the discrete or continuous time domain, or even translated into the distance domain. The following sections elaborate on the constraints set and problem formulation.

\subsection{Dynamic Programming}

Dynamic Programming is a numerical, problem-solving method used for solving complex problems by not having to deal with the complexity of the problem directly. The approach focuses on breaking down the complex problems into a group of simpler subproblems, then solving each of those subproblems just once. The solution of the subproblems are stored in a memory-based data structure such as an array or map. The solutions of the subproblem are each indexed based on the values of their input parameters, as a way to ease the process of looking them up. During computation, if same subproblem was to occur more than once, instead of having to recompute its solution, it simply looks up the previously computed result and as a result, it reduces computation time. Dynamic programming is both a mathematical optimization method and a computer programming method. It provides a methodology to plan trajectories and design controllers and estimators for nonlinear systems $[57,58,59]$. One of the main characteristics of dynamic 
programming approach is the development of a recursive optimization procedure. In this procedure, a solution of the overall problem is built by first solving a simpler one-stage problem. After that, the method proceeds to keep solving one stage problems sequentially until the overall optimum is found. This method requires solving the problem backward as the final stage becomes the first stage. As a result, prior data is built into the procedure in order for it to be able to figure out its path from final point to first point. Alternatively, forward computation is also possible, in which the first stage to be solved is the initial stage of the problem and problems are solved moving forward one stage at a time, until all stages are included. Nonetheless, forward computation cannot be used in problems that include uncertainties [60]. The basis of this optimization procedure is the so-called principle of optimality. The principle of optimality states that an optimal policy has the property that, whatever the current state and decision, the remaining decisions must constitute an optimal policy with regard to the state resulting from the current decision [57]. In this work, the initial and final velocities, the constraints, and the path are fed into the system. As a result, backward computation is used to retrieve the results. Furthermore, it is important to note that DP can also suffer from the curse of dimensionality. This term simply expresses the phenomenon in which the computation and complexity of the problem increase significantly with increase of size. Increase of size can basically refer to the addition of state variable or control inputs. As a result, it is important to find methods that decrease computation time, computation complexity, and need for large discretization. This could allow researchers to address larger problems with more realistic variables.

In order to have a better understanding of DP, it is important to understand its mathematical expression. In order to apply that in relation to control theory, it is important to relate it to the Hamilton-Jacobi-Bellman (HJB) equation. The HJB equation is a partial differential equation that 
yields the value function which gives the minimum cost for a given dynamical system with an associated cost function [61]. A general expression of the dynamic programming use in control theory could be expressed through the problem of finding an admissible control input $u^{*}$ that guides the system : $\quad(t), u(t), t)$ to follow an admissible trajectory $x^{*}$ on time interval $t_{0} \leq t \leq t_{f}$ that minimizes a cost function:

$$
J=h\left(x\left(t_{f}\right), t_{f}\right)+\int_{t_{0}}^{t_{f}} g(x(t), u(t), t) \mathrm{d} t
$$

Where $h$ and $g$ are specified functions. The solution to this problem is an optimal control policy $u^{*}=p(x(t), t)$, which produces an optimal trajectory $x^{*}$ and an optimized loss function $J^{*}$. The loss function follows the fundamental equation of DP expressed through the HJB equation:

$$
-J_{t}^{*}=\min _{\mathbf{u}}\left\{f(x(t), u(t), t)+J_{x}^{* \top} g(x(t), u(t), t)\right\}
$$

in which:

$$
\begin{aligned}
& J_{x}^{*}=\frac{\partial J^{*}}{\partial x}=\left[\begin{array}{llll}
\frac{\partial J^{*}}{\partial x_{1}} & \frac{\partial J^{*}}{\partial x_{2}} & \cdots & \frac{\partial J^{*}}{\partial x_{n}}
\end{array}\right]^{\top} \\
& J_{t}^{*}=\frac{\partial J^{*}}{\partial t}
\end{aligned}
$$

After that, the control input $\boldsymbol{u}$ has to be minimized in terms of $t, x$, and the unknown function $J_{x} *$ and the result is substituted into the HJB equation to get the partial differential equation that is solved with boundary condition expressed by $J\left(t_{f}\right)=h\left(x\left(t_{f}\right), t_{f}\right)$. In order to apply this technique into practice, the continuous process has to be approximated by discretizing the system, which leads to: 


$$
J_{k}^{*}\left(x_{N-k}\right)=\min _{\mathbf{u}_{n-k}}\left\{\hat{f}\left(x_{N-k}, u_{N-k}\right)+J_{k-1}^{*}\left(\hat{g}\left(x_{N-k}, u_{N-k}\right)\right)\right\}
$$

Where $k$ is a stage of $N$-equally spaced discrete time intervals, and where $\hat{f}$ and $\hat{g}$ denote discrete approximations to functions $\boldsymbol{f}$ and $\boldsymbol{g}$. This functional equation is known as the Bellman equation, which can be solved for an exact solution of the discrete approximation of the optimization equation. In simple words, DP follows the assertion that if a-e-g is the optimal path from a to g, then e-g is the optimal path from e to g. DP extends this idea to sequences of decisions which together define an optimal policy and trajectory $[61,62]$. The following section explains the DP formulation for the problem considered in this study.

\subsection{Dynamic Programming Formulation}

In practice, speed restrictions or limits are set throughout roads. To move forward towards implementation, the equations of motion described in Section 2.2 are rewritten in the distance domain. This reformulation is beneficial in handling road data such as grade and speed restrictions as also mentioned in [50]. Thus, the OCP can be reformulated as:

$$
\begin{array}{ll}
\min J & =\int_{0}^{s_{f}}\left(\eta^{q} f_{e}\left(x_{2}, u\right)\right) d s \\
\text { s.t } \quad x_{1}^{\prime} & =\frac{1}{x_{2}}, \\
& x_{2}^{\prime}=\frac{\left(f_{e}\left(x_{2}, u\right)+f_{f}(u)-f_{r}-f_{g}\right)}{M x_{2}}, \\
& x_{1}(0)=0, \quad x_{1}\left(s_{f}\right)=t_{f}, \\
& x_{2}(0)=0, \quad x_{2}\left(s_{f}\right)=0, \\
u & \in\{1,2,3,4,5\},
\end{array}
$$


Where $\eta$ is the motor efficiency and the mode-dependent variable $q$ is defined such that $q=1$ for regenerative-braking and $q=-1$ for propulsion. Note that the equations of motion are expressed in terms of $s$ and hence $x^{\prime}$ is used instead of $\dot{x}$ to represent a derivative with respect to distance rather than time. i.e., $x=\left[x_{1}, x_{2}\right]^{\mathrm{T}}=[t, v]^{\mathrm{T}}$.

To solve the optimal control problem, Dynamic Programming (DP) is used in this study. DP is a powerful numerical technique to determine optimal trajectories explicitly using the Bellman's optimality principle while searching from the final state backward in time. It is well known that DP suffers from computational burden when the number of state and control variables increase, i.e., curse of dimensionality. Nonetheless, DP is still useful to find a global optimum even for nonlinear systems with constraints when the number of variables is small enough [57]. To perform optimization using DP, the equation of motion needs to be expressed in a discrete domain. Discretization inherently introduces numeric errors, which degrades the accuracy of the found solution. To avoid these numerical errors, a careful implementation is important [61]; in this study, discretization steps for time, velocity, and distance are chosen as provided in Table 2.

Table 2 - Discretization in DP Implementation

\begin{tabular}{|c|c||c|c|}
\hline State & $\underline{\text { Symbol }}$ & $\underline{\text { Value }}$ & $\underline{\text { Unit }}$ \\
\hline Velocity & $\Delta v$ & 0.2 & $\mathrm{~m} / \mathrm{s}$ \\
\hline Time & $\Delta t$ & 2 & $s$ \\
\hline Distance & $\Delta s$ & 30 & $m$ \\
\hline
\end{tabular}

For each of the five control modes in (18), a set of equations are used to compute the corresponding acceleration, velocity, and force for a given distance of $\Delta s$ as follows: 
Mode 1, Full Propulsion

$$
\begin{aligned}
& \sim \quad\left\{j \frac{f_{\max }-f_{r}-f_{g}}{M}\right\}, \\
& x_{2, k+1}=\sqrt{x_{2, k}^{2}+2} \tilde{\imath} \\
& f_{e}=M \tau \\
& f_{f}=0
\end{aligned}
$$

Mode 2, Coasting

$$
\begin{aligned}
& \tilde{\imath} \frac{f_{g}}{\imath \boldsymbol{\imath}}, \\
& x_{2, k+1}=\sqrt{x_{2, k}^{2}+2} \tilde{\imath} \\
& f_{e}=0, \\
& f_{f}=0
\end{aligned}
$$

Mode 3, Regenerative Braking

$$
\begin{aligned}
& \left.\sim \quad \underline{u}, \frac{f_{\min }-f_{r}-f_{g}}{M}\right\} \text {, } \\
& x_{2, k+1}=\sqrt{x_{2, k}^{2}+2} \tilde{\imath} \\
& f_{e}=M \tilde{\imath} \\
& f_{f}=0
\end{aligned}
$$

Mode 4, Full Braking

$$
\begin{aligned}
& \tilde{\imath} \\
& x_{2, k+1}=\sqrt{x_{2, k}^{2}+2} \tilde{\imath} \\
& f_{e}=\max \left\{f_{\min }, M \tilde{i}\right\} \text {, } \\
& f_{f}=\min \left\{M_{i} \quad-f_{e}, 0\right\}
\end{aligned}
$$




\section{Mode 5, Cruising}

$$
\begin{aligned}
& \tilde{\imath} \\
& x_{2, k+1}=x_{2, k}, \\
& f_{e}=f_{r}+f_{g}, \\
& f_{f}=0
\end{aligned}
$$

In the equations above, the larger magnitude of the resisting force, $f_{r}$, or grade force, $f_{g}$, in addition to whether the grade is positive or negative, determines whether the force applied to the vehicle is a propulsion force or braking force. Thus, the force in Cruising mode can be either positive or negative depending on the grade and its dominance over the other resisting forces. Note that Mode 4, Full braking mode, involves friction braking in addition to regenerative braking. It is important to clarify that the benefit of this mode is reflected in its use when braking over the regenerative braking limit is needed. This mode would allow the vehicle to respect the speed and time constraints; that is, the vehicle would be allowed to operate at higher speed because rapid deceleration is possible. Additionally, this mode enables the system designer to choose an energy storage device with a smaller capacity that might otherwise limit regenerative braking capability. When computing electrical forces, denoted by $f_{e}^{\text {elec }}$, the motor and generator efficiencies are included, such that:

$$
f_{e}^{\text {elec }}= \begin{cases}\frac{f_{e}}{\eta_{1}} & f_{e} \geq 0 \\ f_{e} \eta_{2} & f_{e}<0\end{cases}
$$

After computing the electrical forces, the total electrical energy consumption is computed as:

$$
E_{\text {total }}^{\text {elec }}=\sum_{k=0}^{N_{f}-1} f_{e, k}^{e l e c} \Delta s
$$


Where $N_{f}$ is the total number of finite horizons in a distance space and $\Delta s$ is the distance discretization as given in Table 2. Note that $\Delta s=30 \mathrm{~m}$ is used after careful simulation proved that the quality of optimal solutions could be kept while computation load is decreased. It can be found that the same discretization length is considered in [50].

\subsection{Case Study}

In this work, a light-weight military ground robot, such as the one in Figure 5, is considered as a target vehicle. As the needs of military vehicles rapidly increase, energy-efficient maneuvering becomes one of the most important requirements among robust performance, mobility and ability to support a variety of electrical loads. Because of the last requirement, electrified powertrains have been receiving attention in all branches of the military for their potential strategic benefits [15]. It should be noted that, although the application considered in this work is to a military ground vehicle, the presented work and approach can be applied to any electrified vehicles.

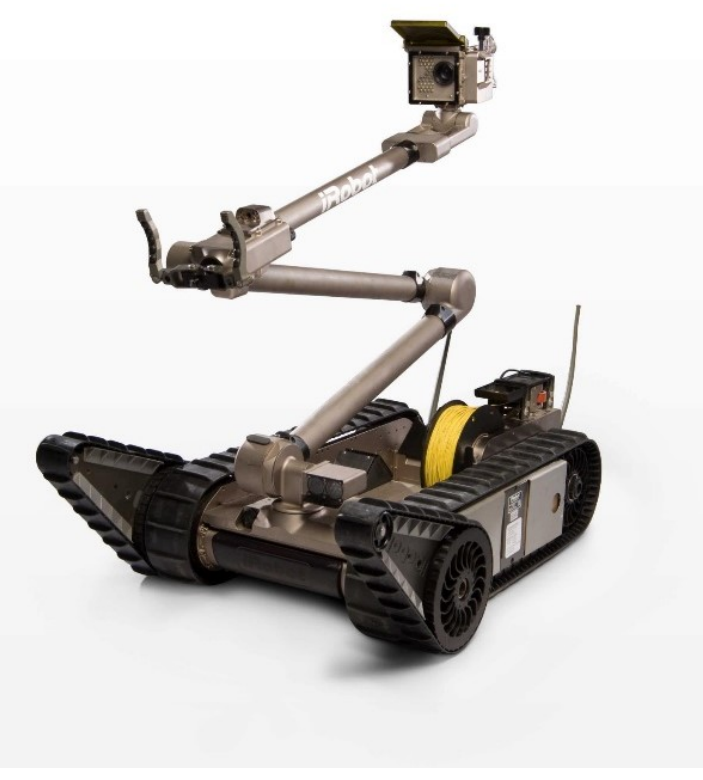

Figure 5 - Sample Model of Considered Lightweight Military Robot [64] 
The Army Ground Vehicle Programs use various drive cycles including time, speed, and grade, for testing and validating new vehicle systems and models. These cycles have traditionally been characterized by run speed, number of stops, and terrain profile. For the sake of powertrain analysis, there have been a number of additional metrics proposed for characterization of such drive cycles in the context of fuel economy evaluation. The drive cycles for ground vehicles focus on running at a constant speed over varied terrain for practical reasons [65]. Thus, two different drive conditions are studied: relatively flat (Convoy) and hilly roads (Churchville B). Specifically, these two drive cycles are considered as the baseline operations when comparing the performance of the optimized speed profile obtained from DP; that is, the total energy consumption and trip time traversing these two cycles are computed from a given set of time, speed, and grade information. Table 3 displays the parameters used in the case study. Note that due to the motor's speed limit, the original Convoy and Churchville B cycles are scaled by a factor of $\underline{0.67}$.

Table 3 - Vehicle Parameters and Constraints

\begin{tabular}{|c|c|c|c|}
\hline Parameter & $\underline{\text { Symbol }}$ & Value & $\underline{\text { Unit }}$ \\
\hline Mass & $M$ & 453.6 & kg \\
\hline A & $\boldsymbol{A}$ & 0.17 & $N$ \\
\hline B & $\boldsymbol{B}$ & 0.06804 & $N s / m$ \\
\hline $\mathrm{C}$ & $C$ & 13.608 & $N s^{2} / m^{2}$ \\
\hline Tire radius & $r$ & 0.2794 & $m$ \\
\hline Final drive ratio & $i_{a}$ & 7.54 & - \\
\hline Speed limit & $v_{\text {lim }}$ & 23 & $m / s$ \\
\hline Max. Acceleration & $\bar{a}$ & 3 & $m / s^{2}$ \\
\hline Min. Acceleration & $\underline{a}$ & -3 & $m / s^{2}$ \\
\hline Motor Efficiency & $\eta_{1}$ & 0.95 & - \\
\hline Generator Efficiency & $\eta_{2}$ & 0.88 & - \\
\hline
\end{tabular}




\section{Non-hilly environment - Convoy cycle}

The Convoy Cycle, shown in Figure 6, has some deviation in speed and a small variation in grade.

From the cycle and parameters in Table 3, the resisting force, $f_{r}$, the grade force, $f_{g}$, and the acceleration required to operate the robot can be computed.

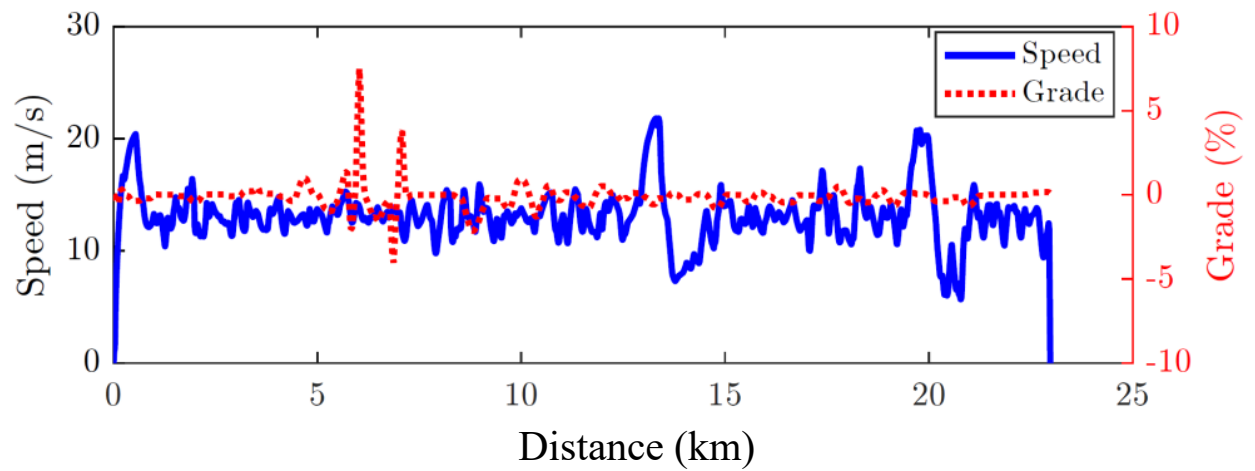

Figure 6 - Baseline Military Drive Cycle: Convoy Cycle

The total electrical energy consumed from the Convoy cycle was calculated to be $1.06 \mathrm{MJ}$, which represents the baseline value for a non-hilly operation. Figure 7 displays the results of total energy consumption for different trip durations, which clearly shows a trade-off between energy consumption and trip time. Particularly, when the trip time is set to be similar to that of the baseline, the total electrical energy consumed to traverse the optimal speed profile obtained by DP is $21 \%$ less, i.e., $0.84 \mathrm{MJ}$. When the amount of energy consumed is set to be similar to that of the baseline Convoy cycle, a 14\% reduction in trip time is achieved. 


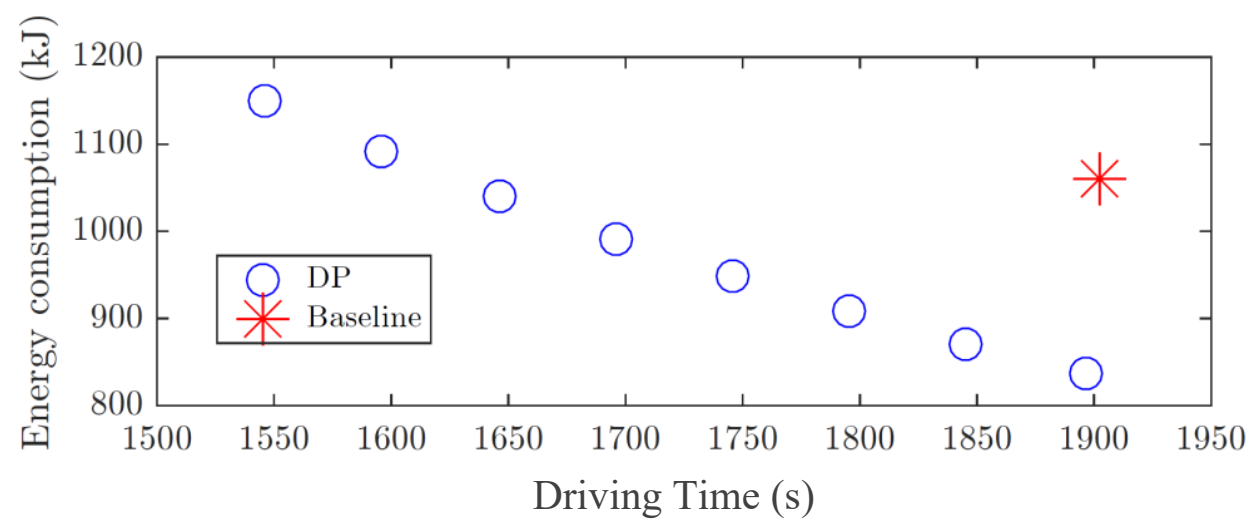

Figure 7 - Tradeoffs Between Energy Consumption and Trip Time for Convoy Cycle

Figure 8 shows the speed profiles over two different trip times in addition to the baseline Convoy cycle. With the speed limit set at $23 \mathrm{~m} / \mathrm{s}$ and with a non-hilly road, the vehicle does not show frequent mode changes, but a stable cruising operation that allows the vehicle to stop within the set trip time. Additionally, for the same distance but with a longer trip time, the vehicle tends to decrease its speed, which obviously reduces the energy consumption. The propulsion and cruising that the vehicle executes are due to the little deviation in grade that allows the vehicle to increase its speed at downhills without enduring additional energy loss. The regenerative braking is used at the end of the trip to stop in accordance with the final velocity constraint. 


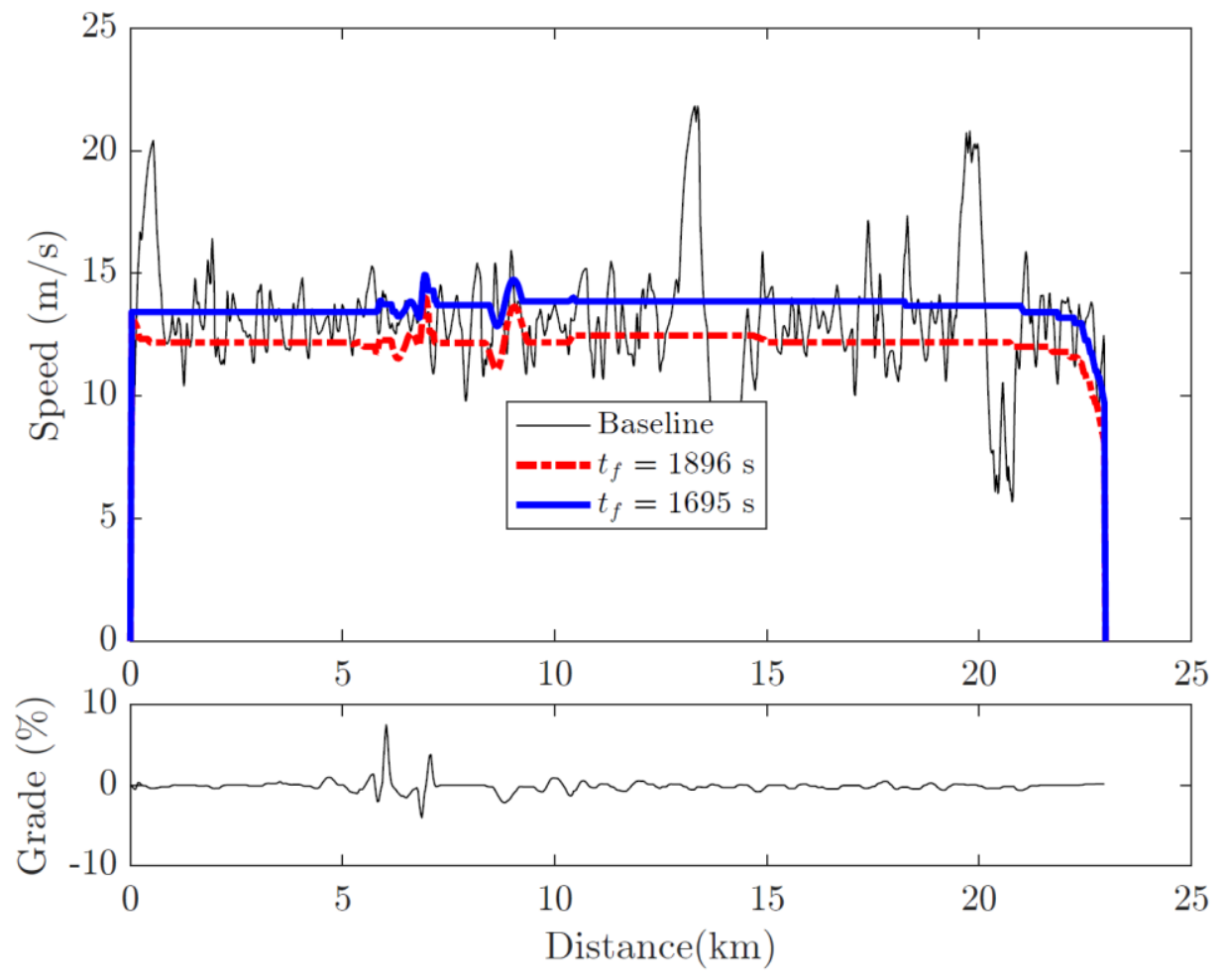

Figure 8 - Comparison of Speed Profiles Over Convoy Cycle

Rugged hilly environment - Churchville B Cycle

Unlike the Convoy cycle, the Churchville B cycle provides constant velocity throughout the trip.

However, this cycle has a more discernible grade change that highlights the performance benefits of velocity optimization over a more irregular course or terrain that has steeper grades. Figure 9 displays the grade changes and constant velocity of the cycle.

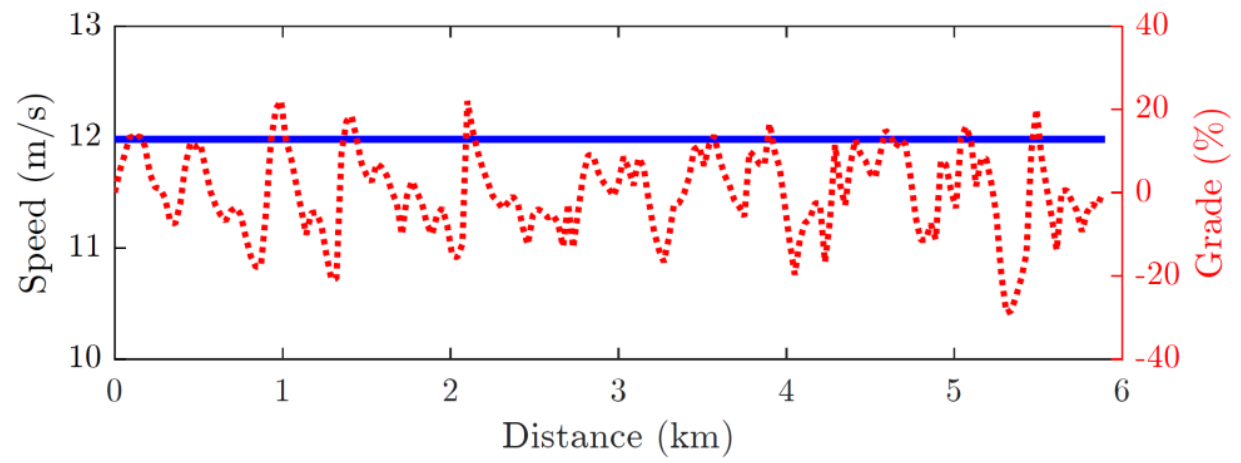

Figure 9 - Baseline Military Drive Cycle: Churchville B Cycle 
The total electrical energy consumed from the Churchville B cycle was calculated to be $0.41 \mathrm{MJ}$, which represents the baseline value for the rugged-hilly operation. Using trip time similar to the baseline, the solution to the OCP shows a $24 \%$ reduction in energy consumption, i.e., $0.31 \mathrm{MJ}$. Moreover, it is observed from Figure 10 that a $24 \%$ reduction in total trip time can be achieved when consuming the same amount of electrical energy as the baseline.

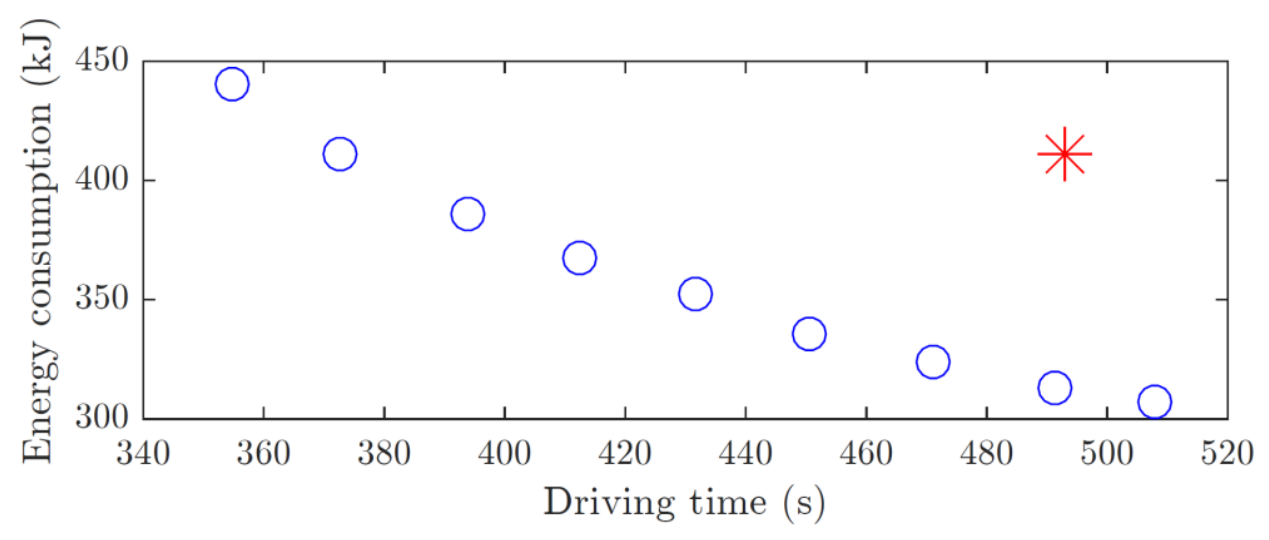

Figure 10 - Tradeoffs Between Energy Consumption and Trip Time for Churchville B Cycle

The results in Figure 11 display the speed profile for Churchville B cycle over two different trip times compared to the baseline. The vehicle exhibits several mode changes as it drives over an uphill and downhill distinctively; in particular, coasting and cruising modes are effectively utilized to minimize energy consumption. As the trip time decreases, the vehicle tends to make better use of increased speed at downhills and uses the propulsion mode more often. In contrast, for a longer trip time, the vehicle tends to use more braking that could benefit in regenerating energy while respecting the time constraint. 


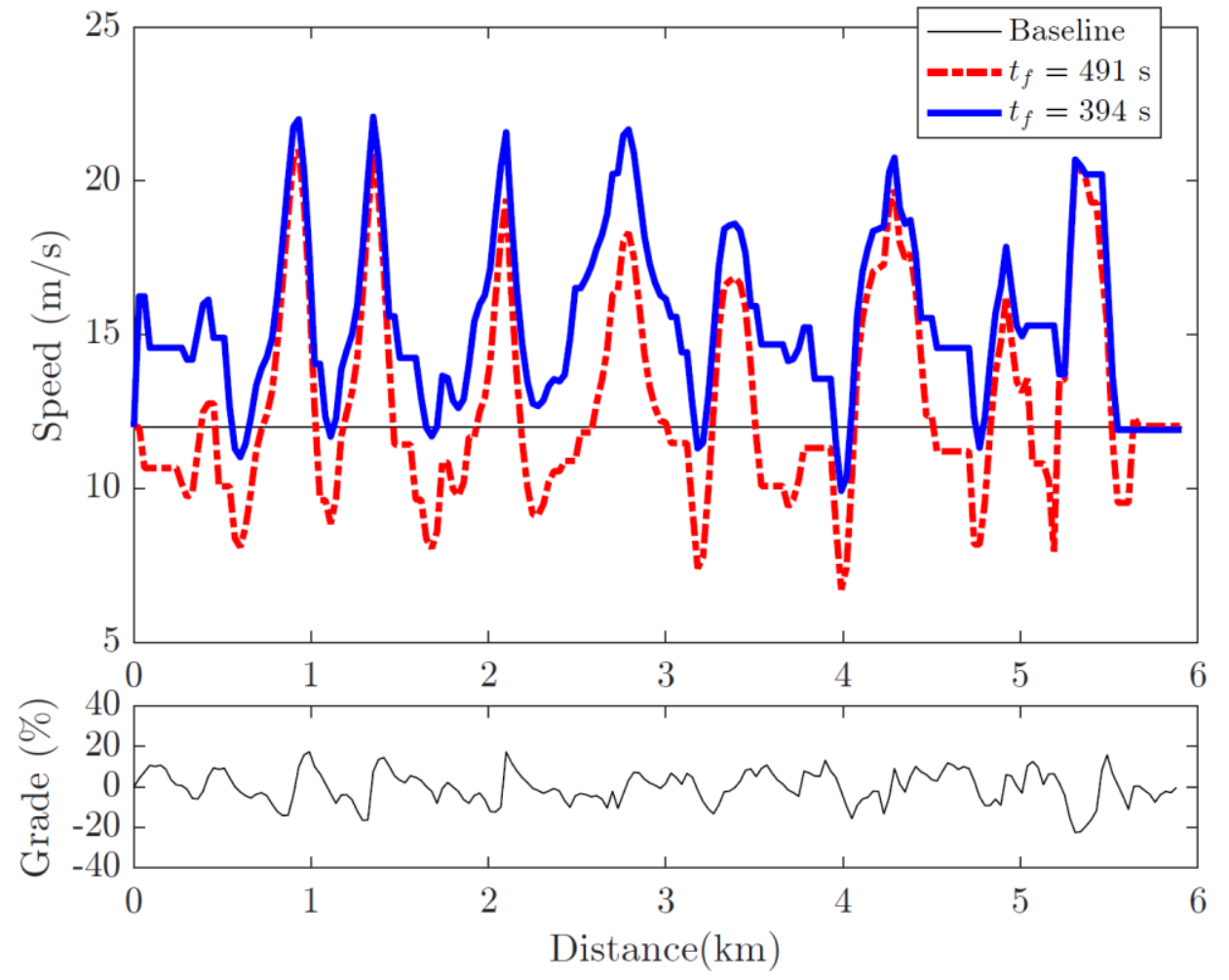

Figure 11 - Comparison of Speed Profiles Over Churchville B Cycle 


\section{Chapter 4: Influence of Powertrain Design on Performance and Control Modes}

\subsection{Impact of Hybridization - Battery Power Capability}

The main aim of the work done in this thesis is to reduce energy consumption of electrified vehicles with various power sources, for instance, fuel-cells and batteries. Sizing those components could affect optimal trajectories. For instance, additional power comes at the cost of increase in battery size, and increased size can result in reduction of energy consumption due to increased regenerative capability. To assess the impact of different battery sizes (or, regenerative power capability) on the energy consumption of the vehicle, a parametric study is conducted by response surface methodology with the following battery power capabilities:

$$
P_{\text {reg }} \in\{10,15,20,25\} \mathrm{kW}
$$

Note that motor power is set at $30 \mathrm{~kW}$, which can be interpreted such that all power sources including fuel-cells and batteries can provide sufficient power to operate the motor over the considered driving cycles. Figure 12 displays a schematic of model of the focused areas of study in this thesis. The vehicle provides control actions that operate the battery or fuel cell to provide the necessary power to the motor that either works to apply propulsion or generation, depending on whether acceleration or deceleration is required. The motor then provides the necessary speed and torque to operate the wheels to move the vehicle. The environment plays a significant role in affecting the performance of the vehicle. The impact comes from speed constraints, road attributes, or road grade that directly affect the operation of the vehicle. An energy model is built based on the information of the energy used, and that data is used by the optimization module in order to 
provide the necessary feedback for the vehicle to operate with the optimal control mode in order to maintain speed and minimize energy consumption. The impact of the battery on the operation is in its ability to provide the necessary power to the motor. In case the battery is unable to provide the required power, fuel cell operates. Nonetheless, the regenerative braking is directly affected by the battery capability. The battery size affects how much regenerative braking can be applied by the vehicle and therefore fuel cell cannot cover up for the battery in this mode.

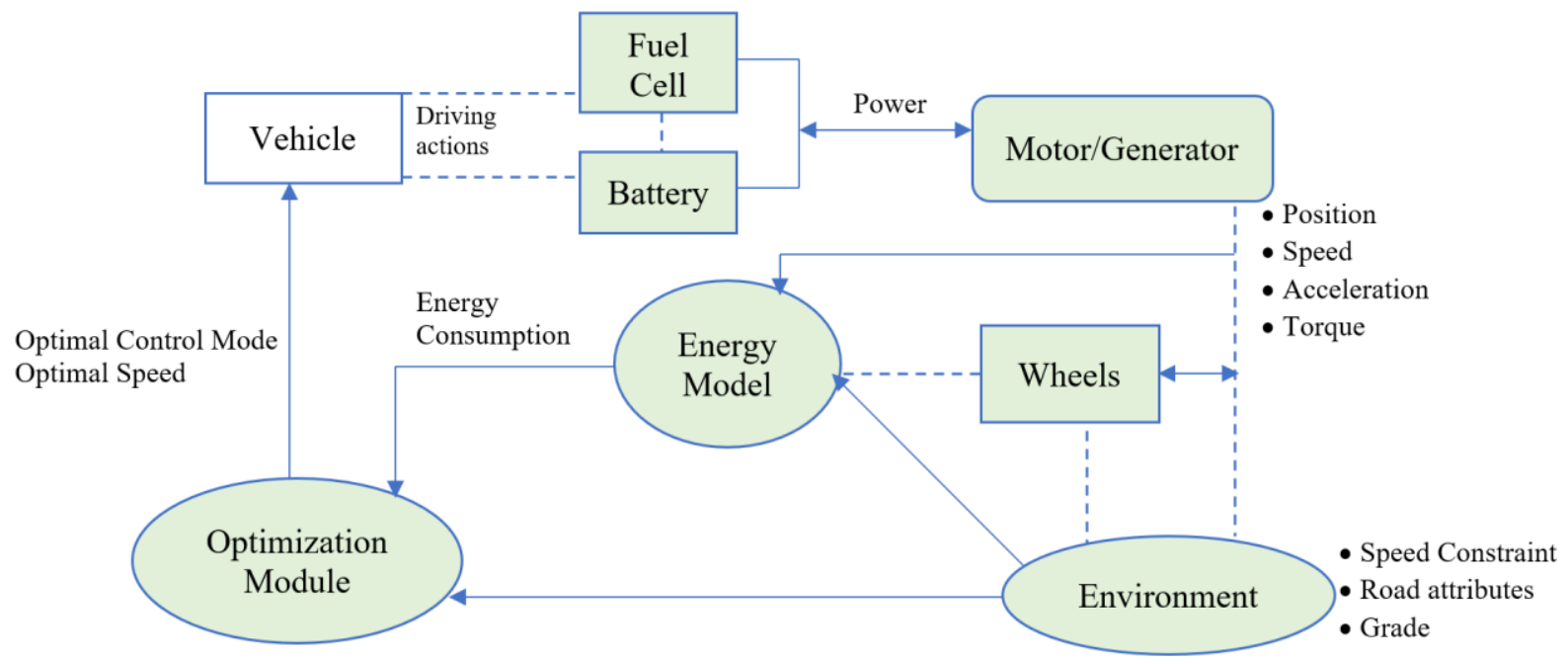

Figure 12 - Schematic of Focused Areas of Study

In this study, it is assumed that the battery energy or state-of-charge (SOC) constraints do not become active throughout the trip. That is, any regenerative braking events following full propulsion do not recuperate more than the energy used for vehicle propulsion. This assumption helps avoid adding the battery SOC dynamics. The inclusion of energy constraint to address long downhill operation will be addressed in future work. As shown in Figure 13, the Convoy cycle reflects no difference in energy consumption for different regenerative power capability ranging between 10 and $25 \mathrm{~kW}$. 


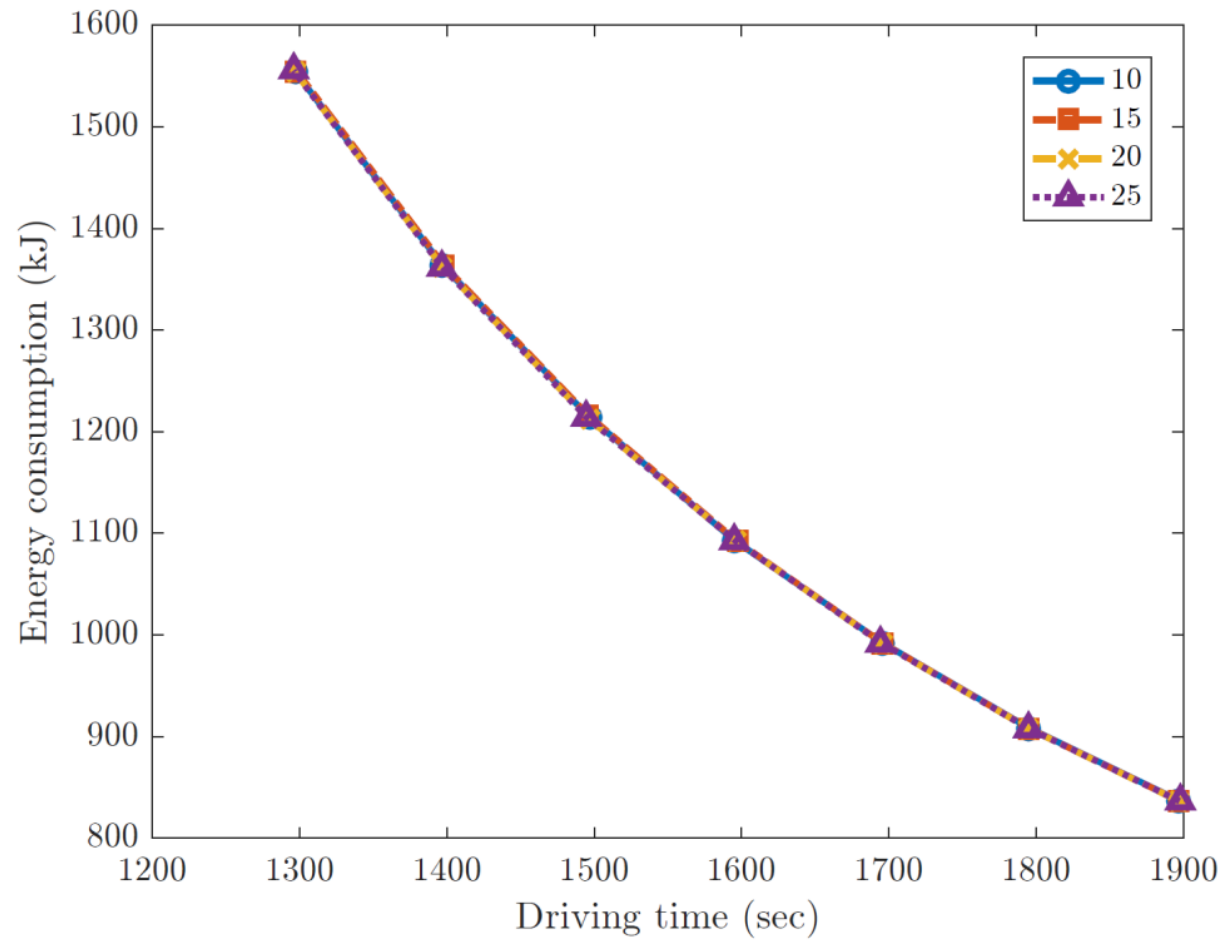

Figure 13 - Influence of Regen-Capability on the Energy Consumption and Driving Time: Convoy

When it comes to the Churchville B cycle, the results, shown in Figure 14, show that as the regenerative power capability increases, energy consumption is decreased; however, as the regenerative power capability reaches $20 \mathrm{~kW}$, there is no further improvement. In other words, the sizing parameter has a saturation point after which increasing the regenerative power capability adds no value to the vehicle. 


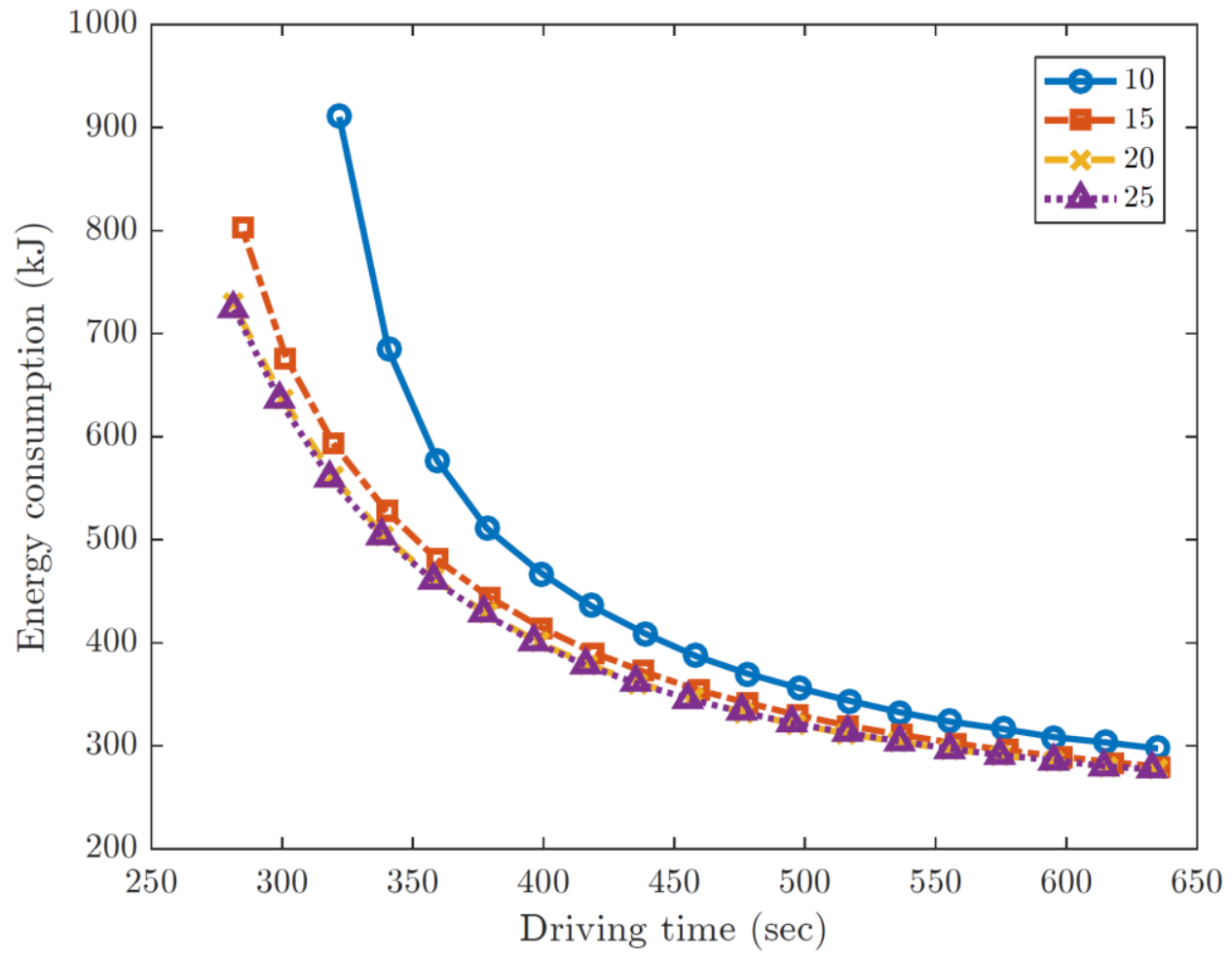

Figure 14 - Influence of Regen-Capability on the Energy Consumption and Driving Time: Churchville B As shown in Figure 15, increasing the regenerative power from $10 \mathrm{~kW}$ to $15 \mathrm{~kW}$ improves the energy consumption by as much as $35 \%$. It can be observed that as the trip time set increases, the impact of resizing the regenerative power decreases to as low as $6 \%$. Moreover, increasing the regenerative power from $15 \mathrm{~kW}$ to $20 \mathrm{~kW}$ has a lower benefit on the energy savings. The maximum energy savings is as high as $6 \%$ and drops to as low as $2 \%$. These results reflect on the idea of the reduced impact of increasing the regenerative power. 


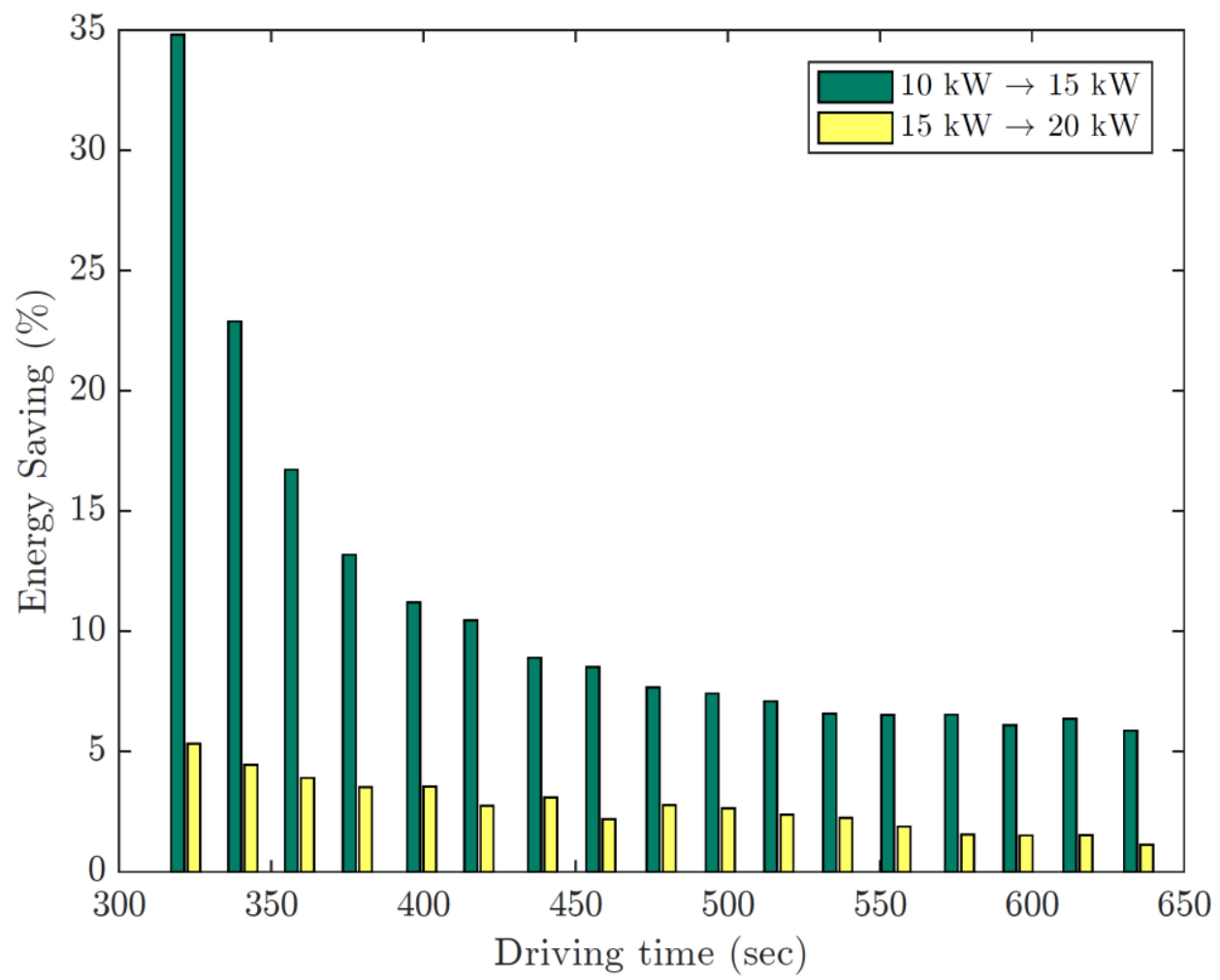

Figure 15 - Percentage Saving in Energy Consumption by Increasing Regen-Capability Over Churchville B Cycle

The vehicle has capability constraints set on it through the power limit and acceleration limit. For instance, due to the deceleration limit set on the vehicle, the regenerative power required from the vehicle would not exceed $25 \mathrm{~kW}$. As a result, increasing the regenerative power of the vehicle would not have a significant impact. Referring to Figure 16, it can be observed that during braking, a majority of the points fall in the low power zones. Furthermore, as the regenerative power is increased from $15 \mathrm{~kW}$ to $20 \mathrm{~kW}$, only 5 points fall outside the $15 \mathrm{~kW}$ domain while 1 point only falls in the $25 \mathrm{~kW}$ domain. Hence, there is no significant impact on the minimization of energy consumption with an increase in regenerative power beyond $20 \mathrm{~kW}$. 


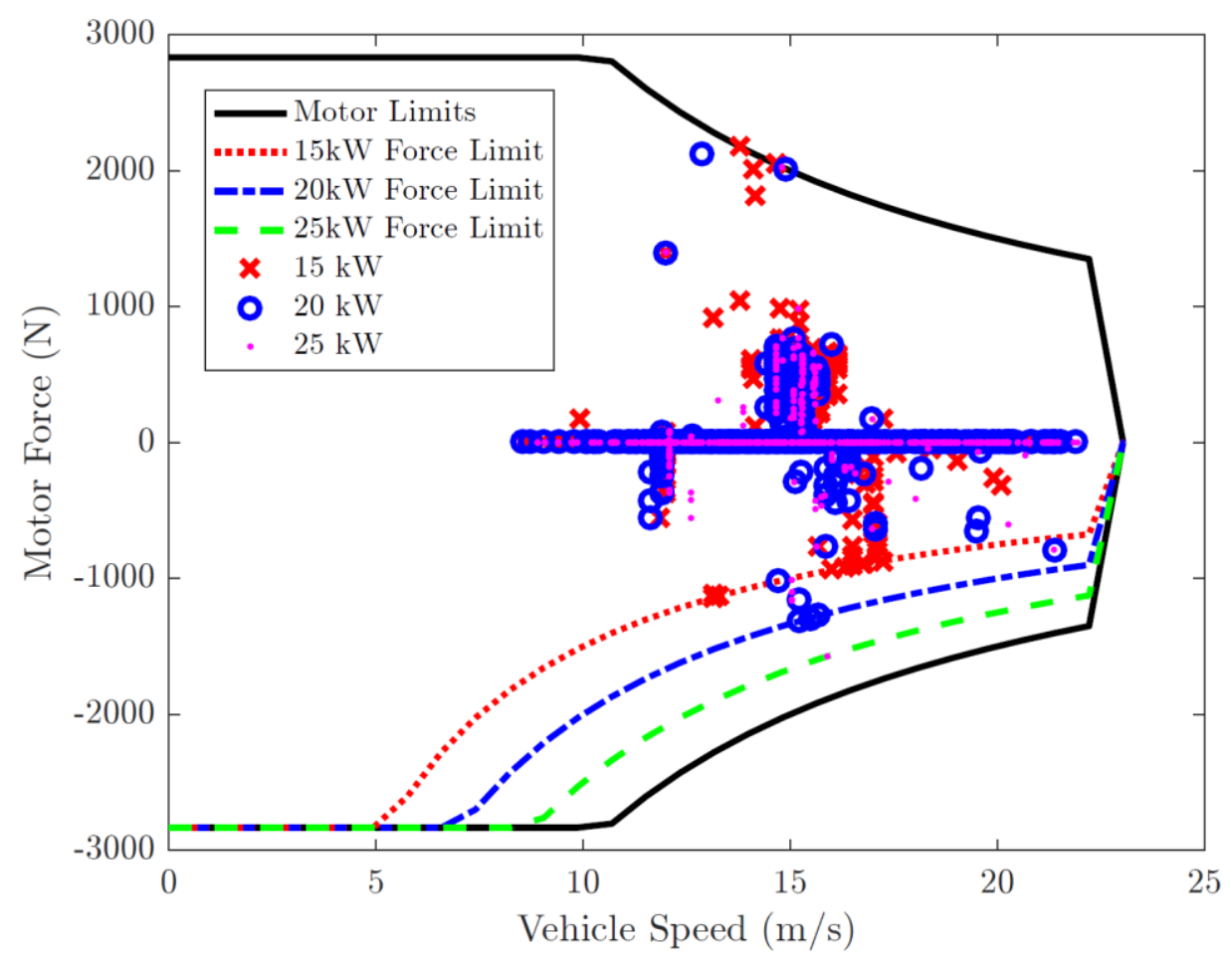

Figure 16 - Comparison of Operating Points for Different Regenerative Braking Capability Over Churchville B Cycle for a Trip Time of 350 Seconds

The results retrieved from the simulations highlight the importance of operational environment in design and control. The hilly Churchville $\mathrm{B}$ cycle allows the vehicle to use coasting and regenerative braking more often at downhills. The use of the regenerative braking mode requires higher regenerative power capabilities of the motor. On the other hand, the Convoy cycle has minimal grade change and requires the regenerative braking mode only at the end of the cycle to stop the vehicle within the time constraint. As a result, there is no significant impact of battery size on energy consumption. Furthermore, increasing the regenerative power capability reflected greater flexibility in decreasing the driving time for a trip. With an increased regenerative braking capability, the vehicle has more flexibility to increase its speed while sticking to the final distance/speed set. 


\subsection{Impact of Control Modes}

Statistical information about control modes over the two driving cycles for different time constraint is shown in Figure 17. It can be observed that the hilly Churchville B terrain exhibits more use of coasting. Driving downhill, the vehicle can use the grade force to its benefit for as long as the vehicle is maintaining the speed limit. In the non-hilly Convoy cycle, the vehicle is focused more on cruising mode. The straight path for the vehicle allows the vehicle to keep the balance of overcoming the resisting forces through cruising. Secondly, increasing trip time increases the use of coasting which is an efficient mode in minimizing energy consumption. Having more time flexibility, the vehicle tends to save energy by exerting no additional forces. Nonetheless, in the Churchville B cycle, the coasting mode is not inevitably increasing with increased trip time. The reason comes down to the importance of the operational environment that plays a significant role in the mode changes that occur. Notably, the constraints of deceleration, acceleration, speed, and distance limit play an important role in selecting the optimal control modes. Lastly, it is evident that as the regenerative power capability increases, the use of the full propulsion and regenerative braking modes decrease. The reason for the decrease in using these modes comes down to the increase in the use of coasting which leads to higher averaged velocity. 


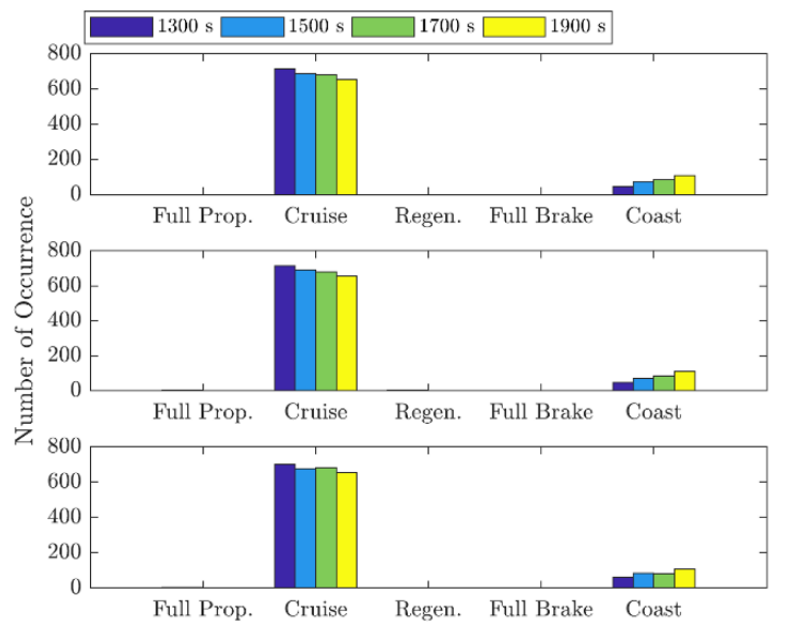

(a) Convoy Cycle

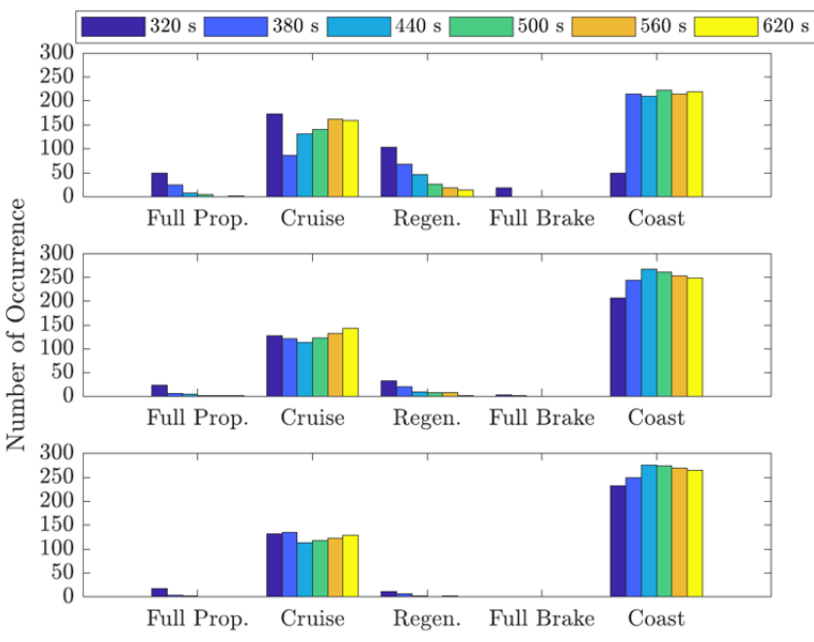

(b) Churchville B Cycle

Figure 17 - Histogram of Control Modes for Different Trip Times with Various Regenerative Capability: $10 \mathrm{~kW}$ (Top); $15 \mathrm{~kW}$ (Middle); And $20 \mathrm{~kW}$ (Bottom)

One of the main advantages of the PMP analysis performed in this paper is the optimization approach using only five modes. The use of full braking mode is advantageous in many ways. Firstly, when the trip time is a constraint, the full braking mode allows the vehicle more flexibility in applying the maximum speed for a longer duration while arriving at the destination with zero velocity within the time set. Although there is a deceleration limit set on the vehicle that might cause the full braking mode not to be beneficial in this case, including the mode is still the proper way to compute a speed profile. Secondly, the full braking mode allows operation with smaller batteries, at times when regenerative braking capability is insufficient. As a result, this would allow more freedom to downsize the battery to compensate for the size of another energy source that could be used to hybridize the vehicle and provide an even higher overall efficiency.

\subsection{Impact of Efficiencies on Control Modes}

As discussed in previous chapters, the optimized speed profile uses different control modes when traversing through a certain distance. The use of the control modes depends solely on minimizing 
the energy consumption of the vehicle. Sections 4.1 and 4.2 describe the effect of regenerative power capability on the use of control modes. However, this section analyses the effect of the motor efficiency on the control modes. Different efficiency values are used ranging from $89 \%$ to $95 \%, \eta_{1}=\{0.89,0.91,0.93,0.95\}$. For the Convoy cycle, as seen in Figures 17 and 18, cruising and coasting modes are the most used modes as they help the vehicle maintain speed with minimal energy consumption. The Convoy cycle's flat route requires minimal mode changes as the vehicle requires propulsion only in the beginning to accelerate the vehicle while it needs regenerative braking only at the end in order to stop the vehicle. Cruising mode is most used in this cycle as it keeps the balance of overcoming the resisting forces. Coasting mode is used with increase in time flexibility as the vehicle tries to save energy by exerting no additional forces. Using the same set of driving time $\{1300 \mathrm{~s}, 1500 \mathrm{~s}, 1700 \mathrm{~s}, 1900 \mathrm{~s}\}$. Figure 18 displays the statistical information of the effect of efficiency on the use of control modes for the Convoy cycle. When increasing the efficiency from $89 \%$ to $91 \%$, the results show no significant impact of efficiency on the control operations. The reason is because in the Convoy cycle, the vehicle uses propulsion only in the beginning to accelerate and uses regenerative braking only at the end to stop the vehicle. Realistically, the increase in efficiency tends to make the vehicle depend more on cruising which is only evident in the increase from $93 \%$ efficiency to $95 \%$. Nonetheless, the decrease that occurs from $89 \%$ to $91 \%$ is only justified with numerical errors from the approach. Analysis is made on the hilly Churchville B cycle in order to create a solid base for justification of results. 


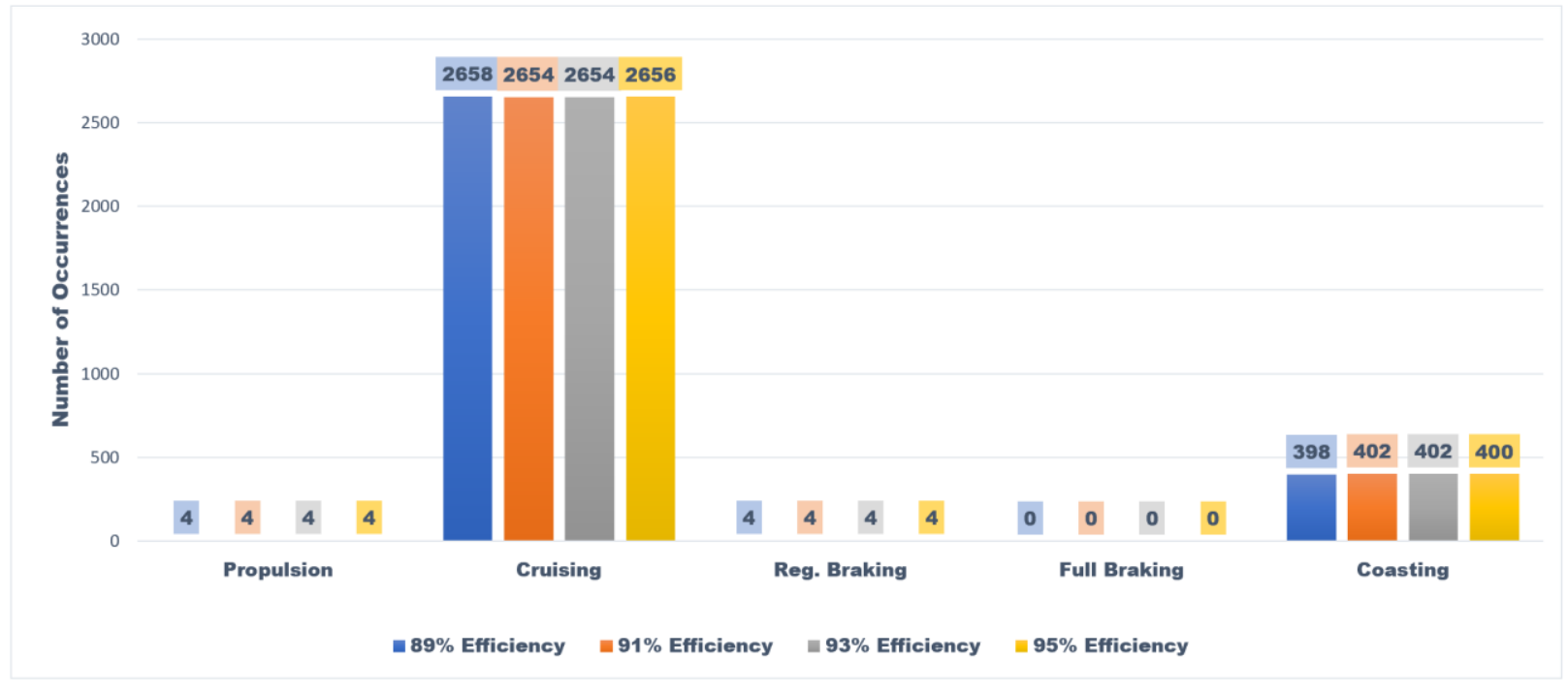

Figure 18 - Impact of Efficiencies on Control Modes for Convoy Cycle

The hilly Churchville B cycle requires multiple mode changes as the vehicle tries to use the environment to its advantage in order to minimize energy consumption. Driving downhill, the vehicle can use the grade force to its benefit for as long as the vehicle is maintaining the speed limit. The impact of changing the efficiency is more evident in this cycle. Figure 19 displays the statistical information of the sum of the number of occurrences of each control mode for all the driving times, but for different efficiencies. Increasing motor efficiency for the hilly cycle shows a great impact on the control modes. As efficiency increases, the use of cruising and regenerative braking modes increases while the use of coasting decreases. At lower efficiencies, the vehicle tends to use coasting mode more often as it requires no exertion of forces and because the cruising mode suffers conversion losses when it switches from generating to motoring and vice versa. Nonetheless, as the efficiency increases, the motoring-generating conversion losses decrease. As a result, the vehicle tends to depend more on cruising and regenerative braking as they help maintain higher speed while respecting the speed, acceleration, deceleration, and time constraints. 
Furthermore, the use of propulsion and full braking do not follow a specific trend as their use is more influenced by the terrain (uphill/downhill).

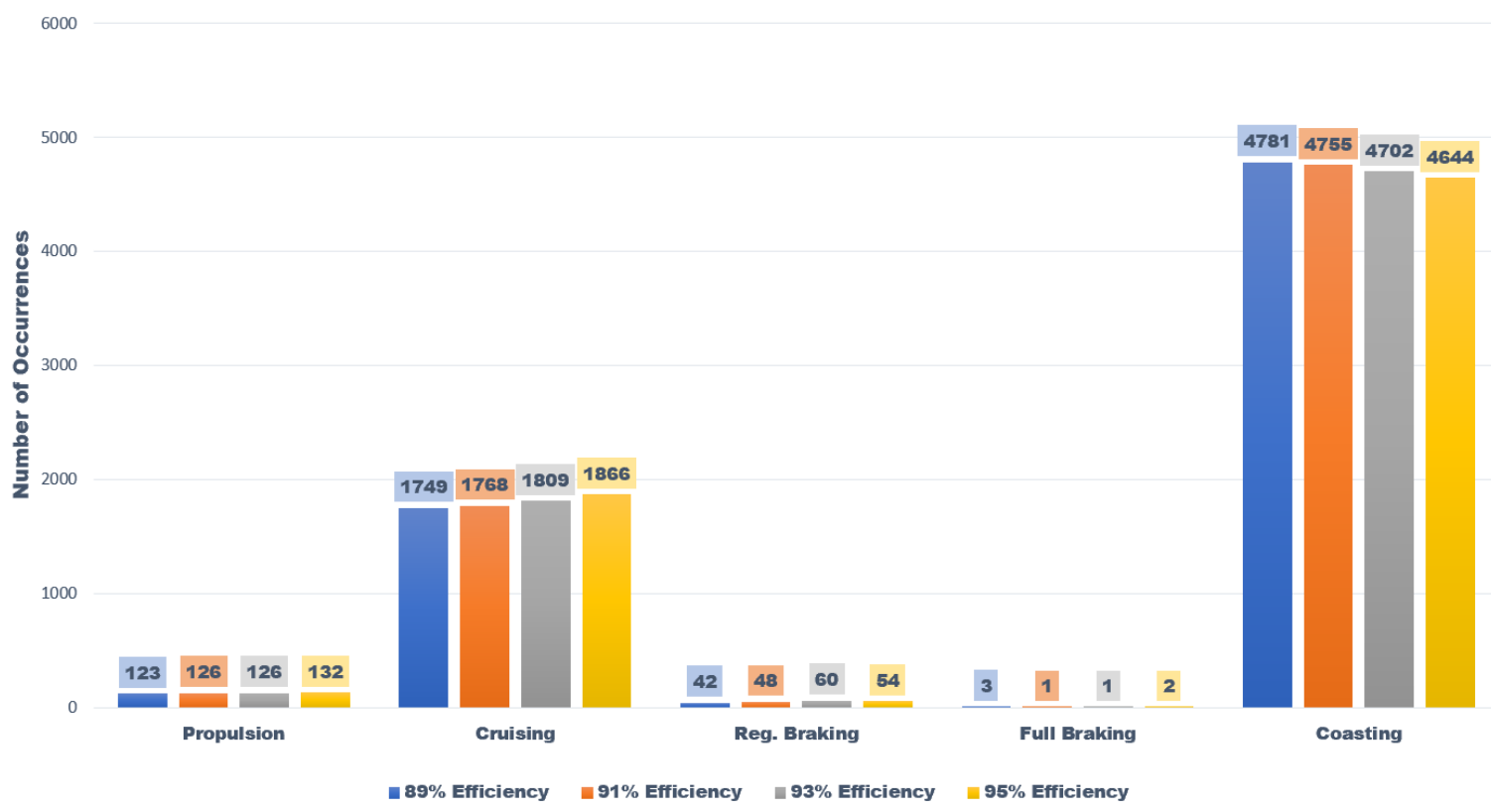

Figure 19 - Impact of Efficiencies on Control Modes for Churchville B Cycle

\subsection{Impact of Using Nonlinear Motor Map}

For the work done in the preceding chapters, the motor and generator efficiencies were assumed to be constant values, $95 \%$ and $88 \%$ respectively. The efficiency of an electric motor is generally nonlinear. Nonetheless, the efficiency is relatively constant above certain force or torque levels covering nominal operating range. In this section, the impact of using a nonlinear motor map instead of the constant value will be investigated. Different approaches are used to retrieve the result of the energy consumed throughout the trip for both the Convoy and Churchville B cycles. Firstly, different runs are performed to compute the energy consumption at different times, using constant motor efficiency values ranging from $89 \%$ to $95 \%$ and constant generator efficiency of $88 \%$. After that, a nonlinear motor map that depends on the speed profile, and mechanical forces is used to compute the energy consumption for the same trips after running the simulations. These 
results provide the suboptimal solution of the energy consumption using nonlinear efficiency. Lastly, the optimization problem is simulated with nonlinear efficiency that is computed throughout the DP computation. The velocity and mechanical forces that are computed for every discrete point are used to retrieve the efficiency value that is used to calculate the energy consumption. The results of this run ensure global optimality. Figure 20 displays the motor efficiency map. At different operating points, the efficiency ranges from $55 \%$ to $96 \%$ with an average value of $90 \%$. Higher efficiency dominates area in which speed of the vehicle is around $8.5 \mathrm{~m} / \mathrm{s}$. Note that the maximum speed constraint set is $23 \mathrm{~m} / \mathrm{s}$.

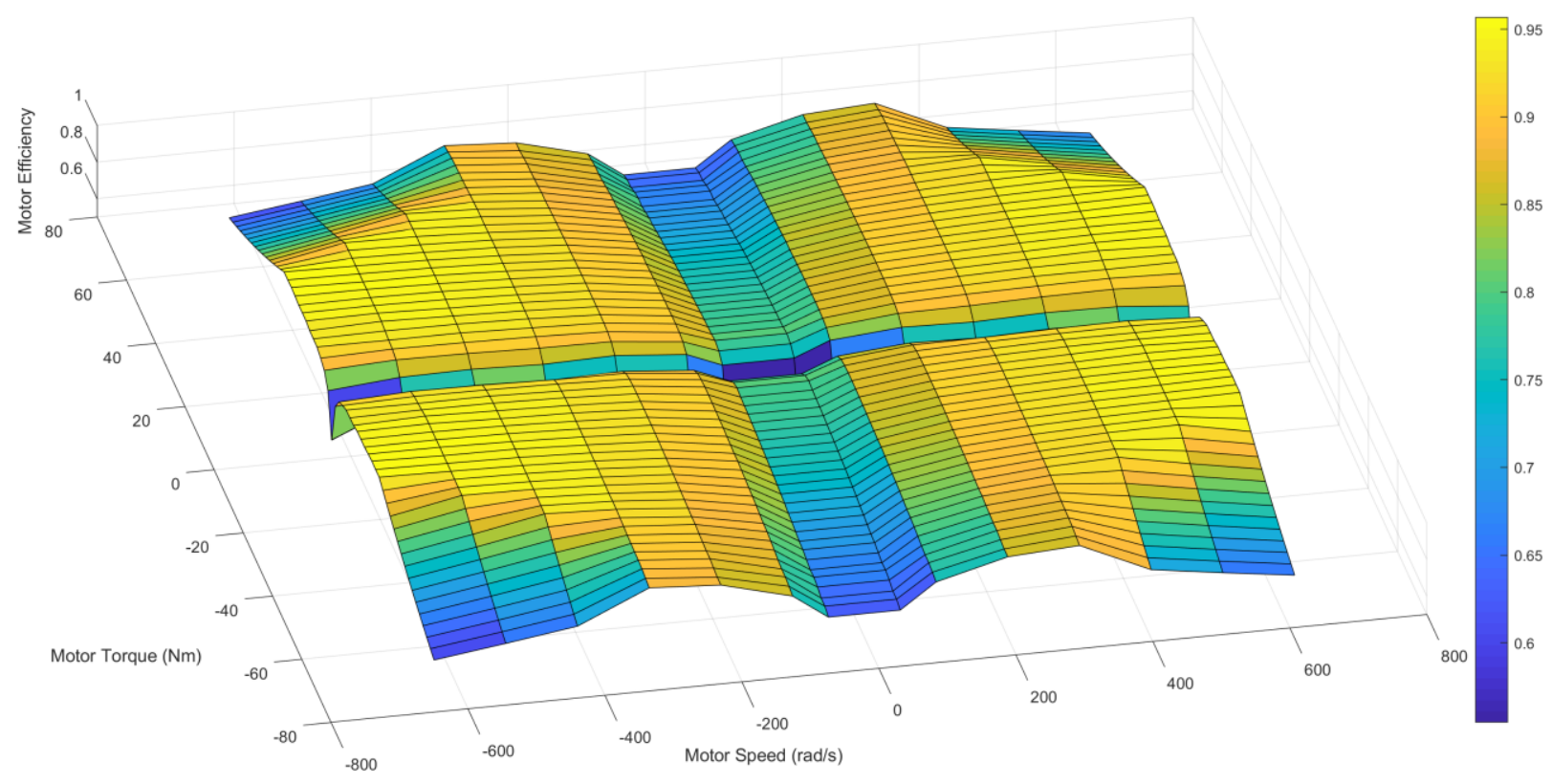

Figure 20 - Motor Efficiency Map

\section{Convoy Cycle}

Operating over a flat route generates different results than operation over a hilly terrain. The terrain plays a significant role in affecting the speed and control modes. For the flat Convoy cycle, Figure 21 displays the results of the energy consumption computed from the suboptimal results that are retrieved after DP computation for constant efficiency values of $89 \%$ and $95 \%$. Moreover, the figure compares these results to those computed from the global optimal approach. As seen, 
the global optimal approach yields lower energy consumption results at all points. This confirms the conjecture that global optimal results of energy consumption yield lower values than suboptimal results. Note that the cost function used in the computation depends on the electric forces of the vehicle. As a result, when energy consumption uses nonlinear efficiency after computation (suboptimal), the velocity and forces would be retrieved based on the cost function that used constant efficiency. On the other hand, when using nonlinear efficiency throughout the DP computation, the cost function would be affected directly by the nonlinearity. As a result, the global optimal ensures more reliable results. Nonetheless, the values do not show a significant difference, and both follow the same trend, confirming the usefulness of both approaches. Moreover, the nonlinear efficiency suboptimal results retrieved after computation of constant efficiencies of $89 \%$ and $95 \%$ shows similar values. This proves that the suboptimal approach is reliable and is actually taking consistent consideration of the efficiency changes regardless of the results computed from different constant efficiencies.

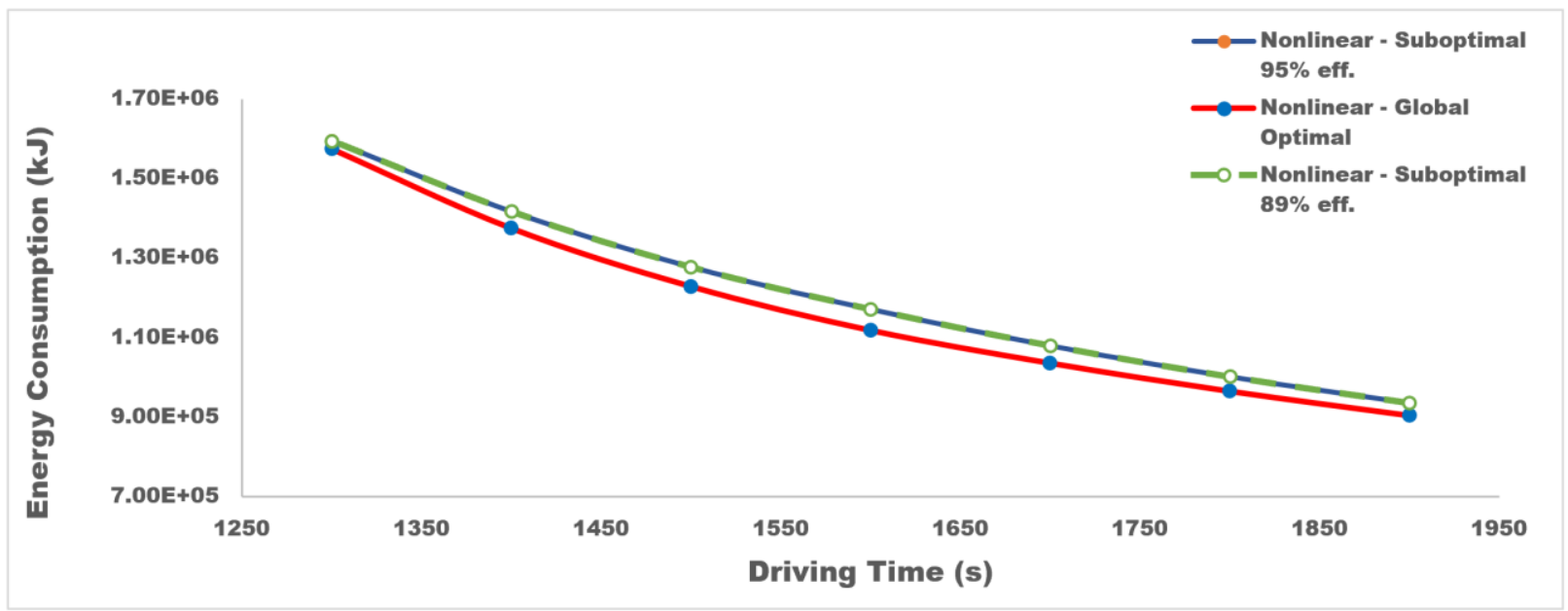

Figure 21 - Sensitivity Comparison between Different DP Approaches for the Convoy Cycle

When it comes to the speed profile, the change in efficiencies and change in approach play a significant role in the control modes and speeds over different discrete points. Figure 22 displays the speed profile and efficiency plot for the Convoy cycle for global optimal results of nonlinear 
efficiency and constant efficiencies of $95 \%$ and $89 \%$. In the global optimal speed profile, the vehicle displays operation of Pulse and Glide (PnG). PnG, also known as artificial glide, is one of the most typical economic driving strategies, which is fitting for proper roads in the plains or with a slope less than $2 \%$ [66]. In this operation, the vehicle accelerates steadily to a higher speed, then the vehicle stops exerting forces and keeps coasting or gliding as a result of the vehicle's kinetic energy stored in the period of acceleration. As the speed drops below $80 \%$ of the desired, high speed, the vehicle accelerates steadily again, and the process keeps repeating throughout the trip. When gliding, the motor idles and stops exerting any energy, resulting in reduction of energy consumption. Combining this operation with the cruise technology that controls the speed, provides a good approach for eco-driving $[67,68]$. PnG operation allows the motor to operate at higher efficiency in pulse while in glide, although the efficiency drops, the coasting mode used prevents conversion losses and allows the vehicle to operate at a higher over efficiency throughout the trip.

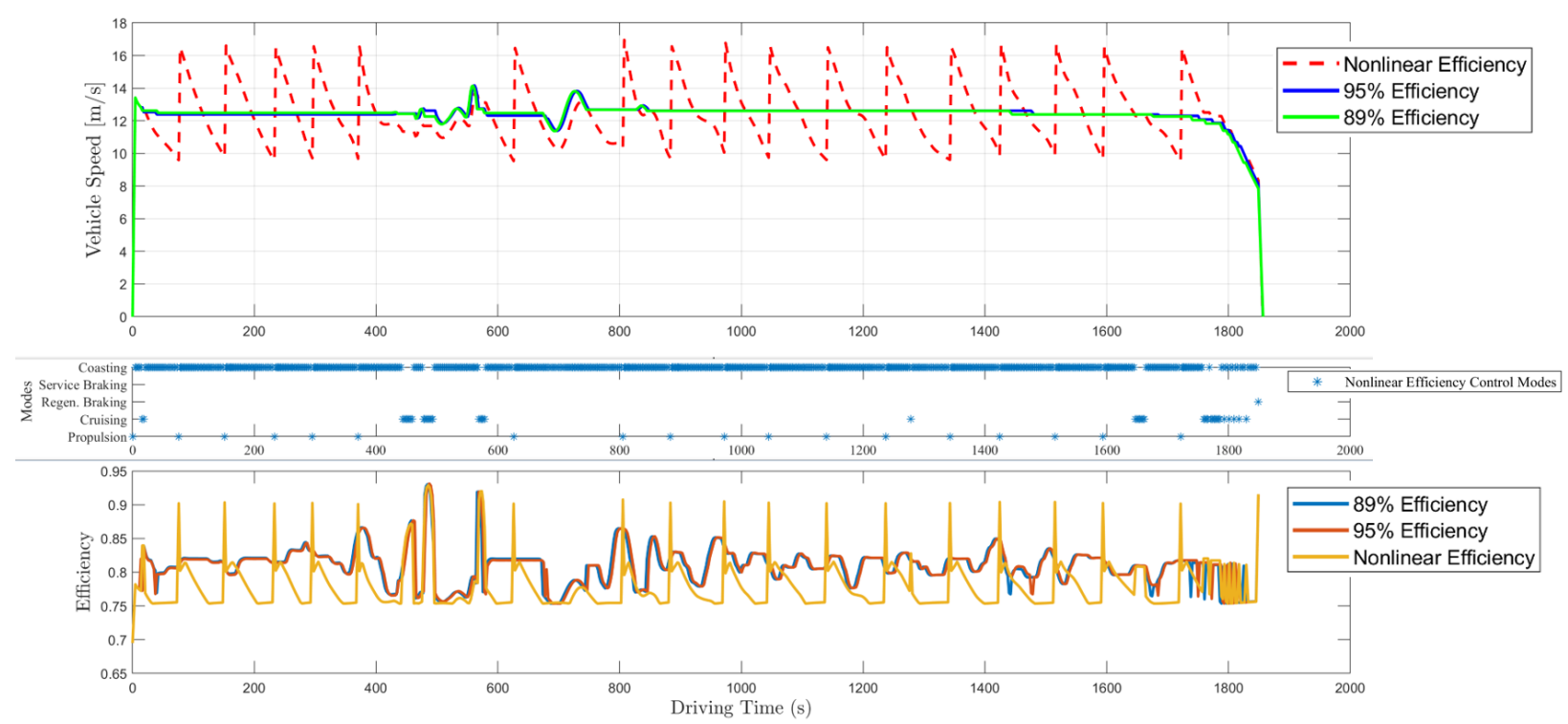

Figure 22 - Speed Profile (top), and Efficiency Plot (bottom) Using Constant and Nonlinear motor efficiency with Emphasis on Control Modes for the nonlinear efficiency results (middle) over the Convoy Cycle 
When it comes to the control mode changes that occur in comparison between the constant efficiencies approach and nonlinear efficiency approach, Figure 23 displays the differences in results. As can be seen, there is a significant increase of the use of propulsion and coasting, and a sharp decrease in the use of cruising mode. The reason comes back to the idea of Pulse and gliding that causes the vehicle to operate efficiently by applying acceleration then gliding (coasting) until the speed drop below $80 \%$ of the efficient speed. Cruising mode is used in this case in order to maintain constant speed for some time.

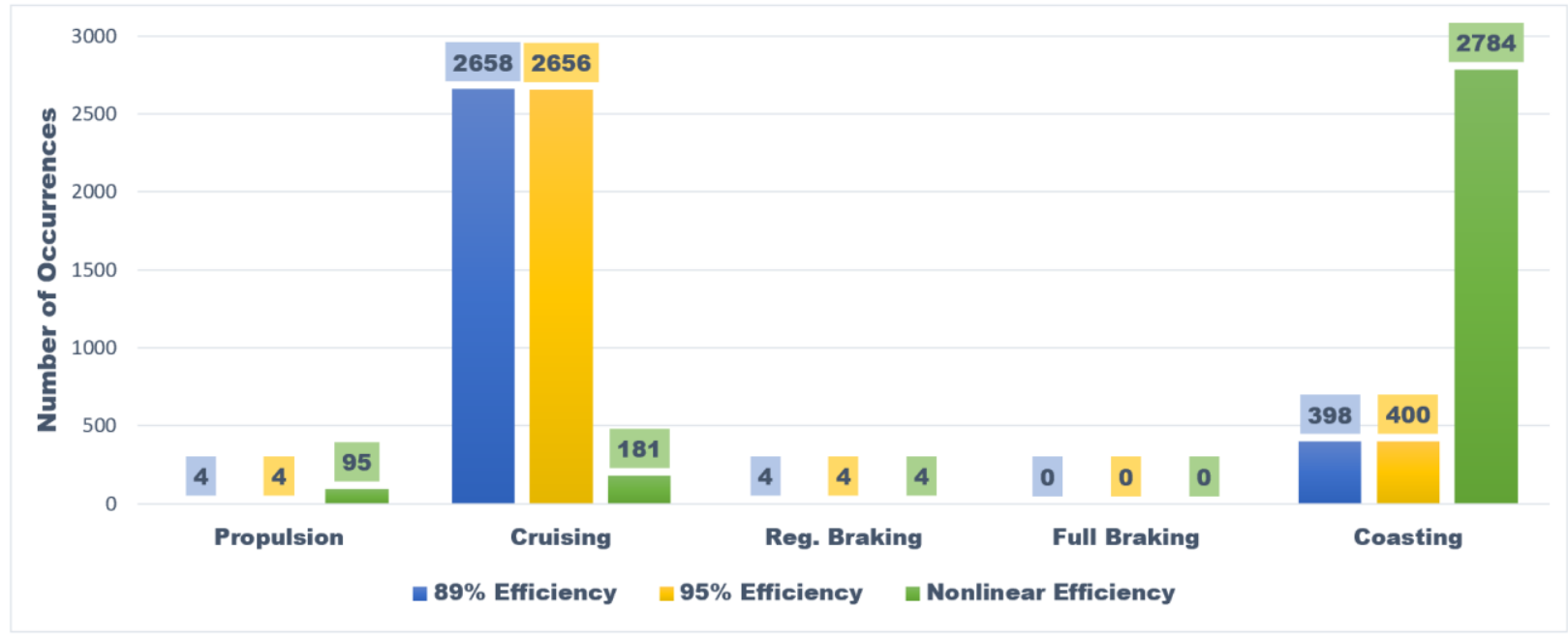

Figure 23 - Control Modes Comparison Between Nonlinear Efficiency Approach and Constant Efficiencies for Convoy Cycle

After extracting the results of the impact of nonlinear efficiency on the control modes, a comparison is conducted to study the impact of time on the control modes for the nonlinear efficiency. Figure 24 displays the results of the analysis compared to the results for constant efficiencies similar to those compared in Figure 17. As seen, the difference is not only in the use of control modes throughout the trip but also in the behavior of the control modes over time. As previously discussed, increase in time for the Convoy cycle for constant efficiencies results in an increase in the use of coasting mode and decrease in the use of cruising mode as the vehicle uses the flexibility in time to exert less forces. Nonetheless, in the nonlinear efficiency, due to the pulse 
and glide operation, as time flexibility increases, the vehicle tends to use cruising mode more often. This is because the vehicle finds the operation of constant speed more efficient than the application of acceleration and coasting over a longer period of time. Afterall, propulsion consumes more energy than cruising and therefore, with increase in time, cruising is used more.

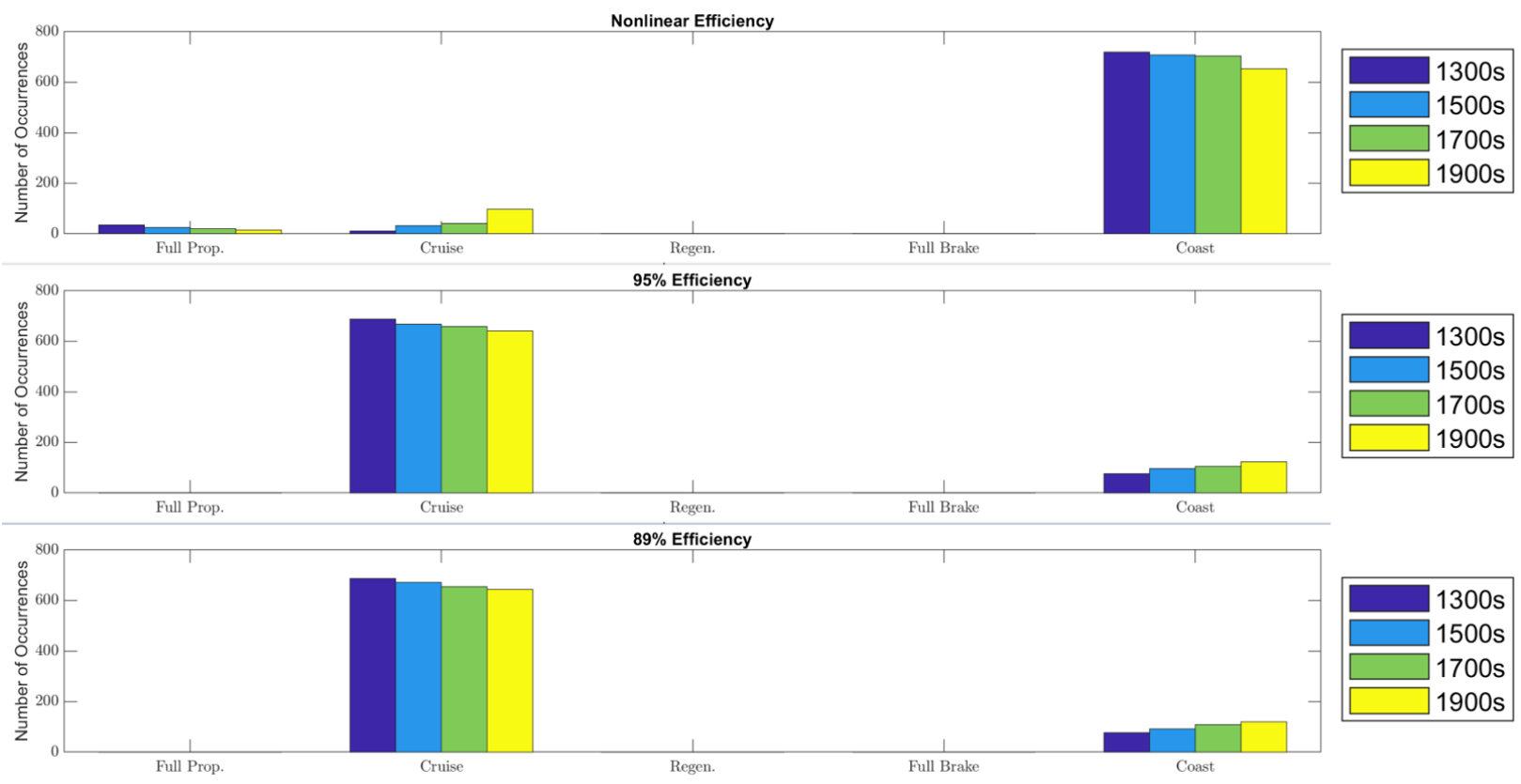

Figure 24 - Histogram Comparing Control Modes of Nonlinear Efficiency vs. Constant Efficiencies Over Different Times for Convoy Cycle

\section{Churchville B Cycle}

The same approaches are applied to the Churchville B cycle to provide a stronger base for analyses and to study the impact of the environment on the consistency of results. Figure 25 displays the sensitivity comparison for the Churchville B cycle. As seen, the difference between global optimal results and suboptimal results follows similar trend to that in the Convoy cycle. Nonetheless, there are small variations in the suboptimal results over different efficiencies. The grade changes that occur in the hilly route cause variations in the control modes used in the two efficiencies. These controls modes variations play a role in the energy consumed at some points. Nonetheless, the overall energy consumption is still similar, and the approach is still reliable and consistent. 


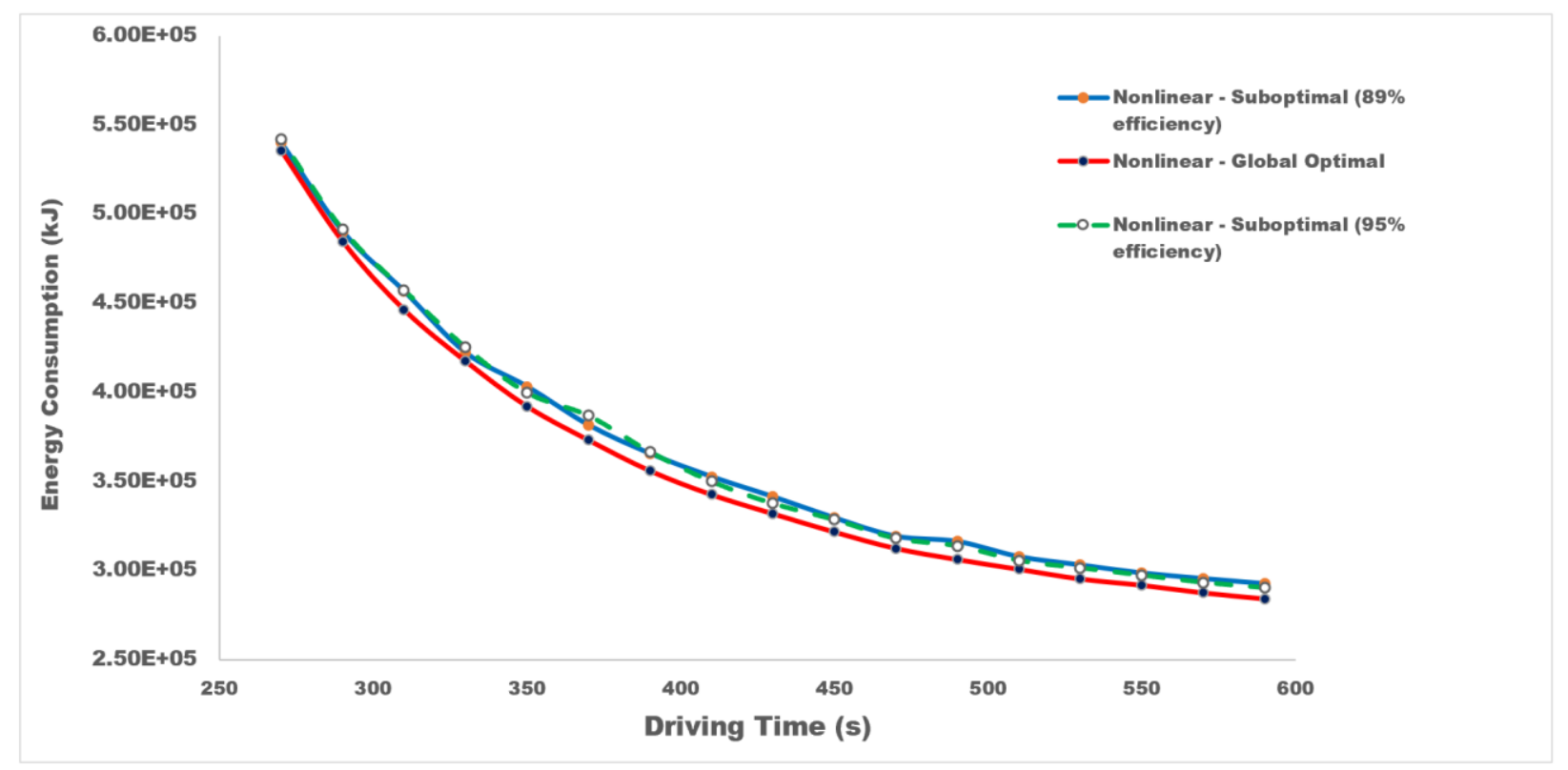

Figure 25 - Sensitivity Comparison between Different DP Approaches for the Churchville B Cycle

When it comes to the speed profile, the impact of the use of the different approaches is also conducted for the hilly Churchville cycle. Figure 26 displays the speed profile for global optimal results of nonlinear efficiency and constant efficiencies of $95 \%$ and $89 \%$. Unlike the Convoy cycle, the vehicle maintains similar trends but at different speeds that result from the use of slightly different control modes over different discrete distances.

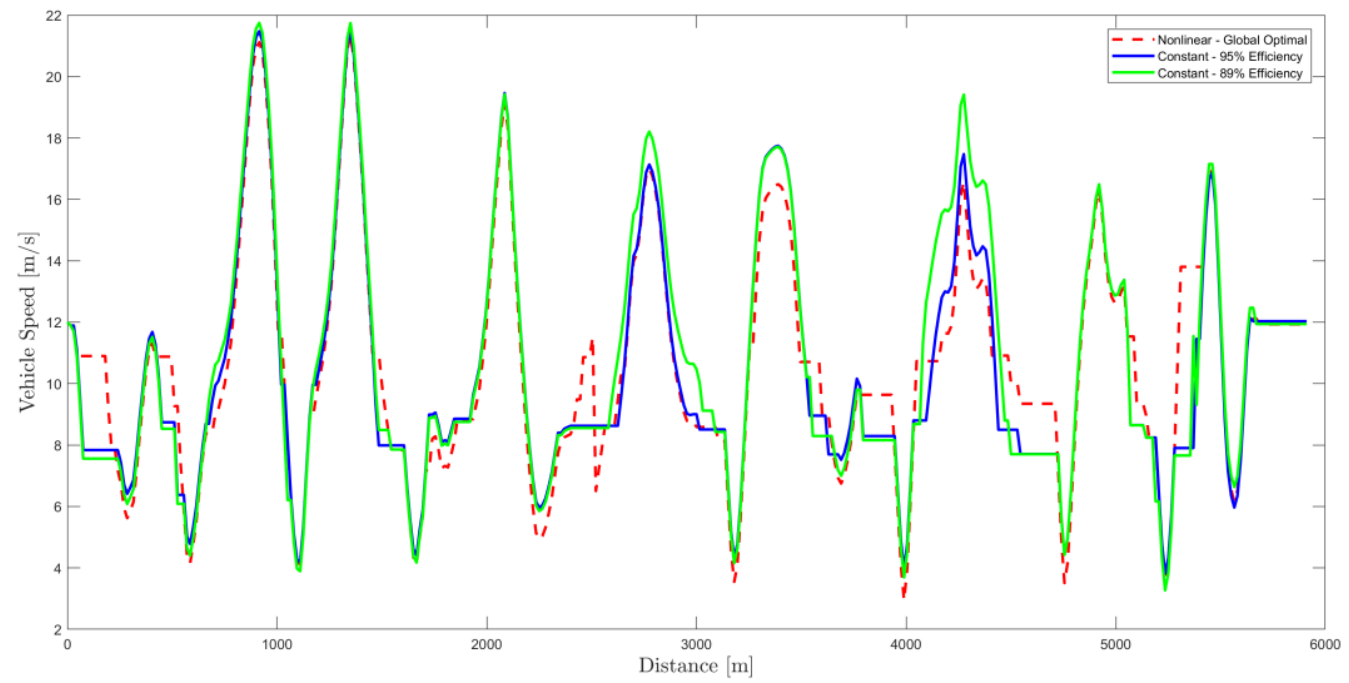

Figure 26 - Speed Profile Using Constant and Nonlinear motor map for the Churchville B Cycle 
In order to have a deeper understanding of the difference in approaches, the changes in control modes needs to be analyzed. Figure 27 displays the overall use of control modes for the different approaches. The simulation trip times used are from 590 seconds to 270 seconds with 20 second decrementation. The control modes over each of the trip times are added together to yield the total use of each mode. As seen, the changes in modes are not significant and they complement the results in the speed profile. For the nonlinear efficiency, the vehicle depends more on coasting and cruising throughout the hilly terrain, and less on propulsion and braking. At points in which the vehicle operates at high efficiency, cruising mode would be utilized more, while at downgrades, coasting mode proves to be the most efficient control mode. The environment still has the biggest impact in the Churchville B cycle.

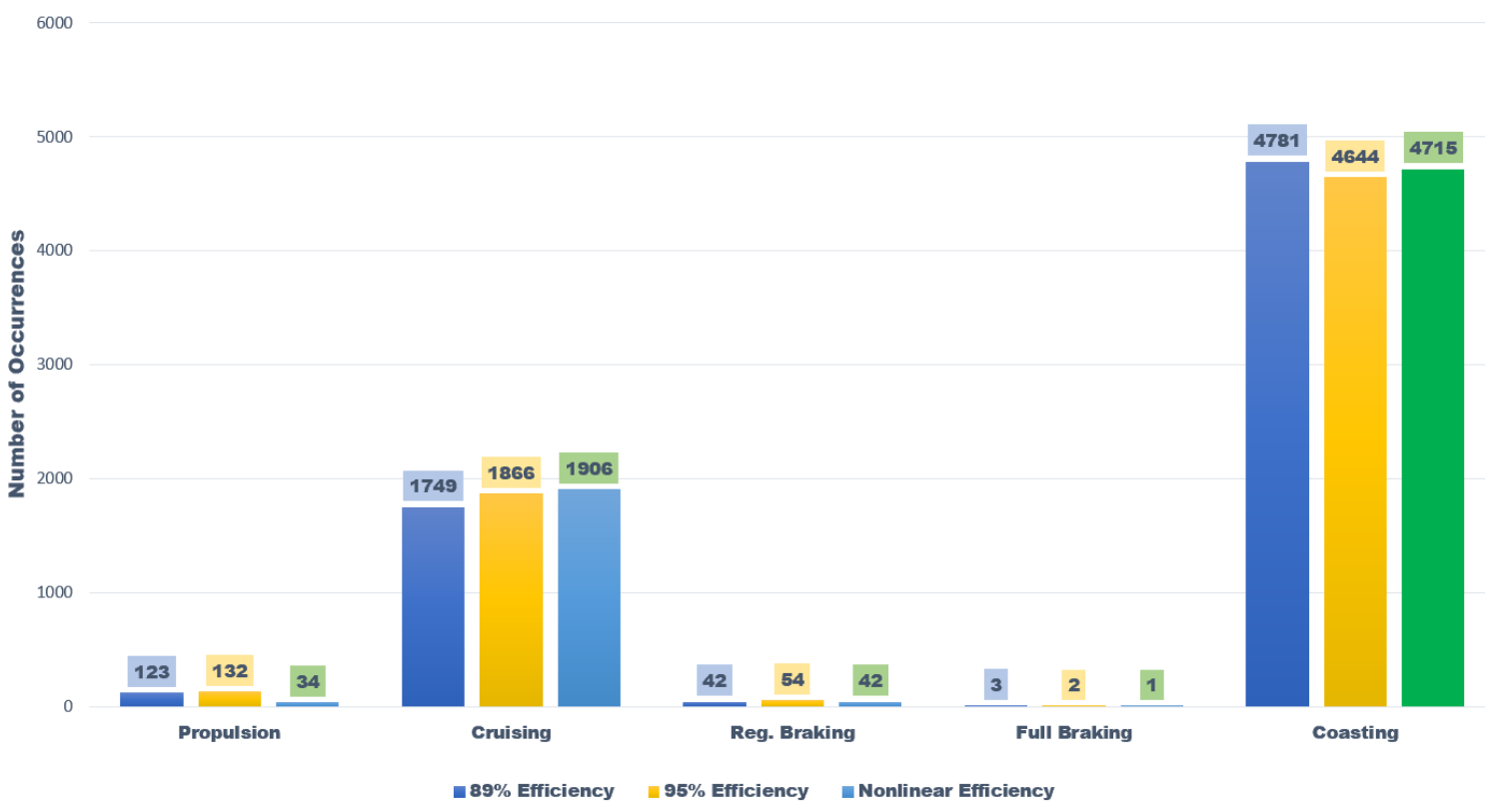

Figure 27 - Control Modes Comparison Between Nonlinear Efficiency Approach and Constant Efficiencies for Churchville B

After extracting the results of the impact of nonlinear efficiency on the control modes, it is important to study the impact of time on the control modes for the nonlinear efficiency over the Churchville B cycle. Figure 28 displays the results of the comparison. As seen in the results over 
increased time, the approaches do not follow a certain trend with increase of time. The difference between the control modes in the nonlinear efficiency approach is slightly different from that in the constant approach. The reason of the difference comes down to the value of the efficiency at a certain point, and the impact of the environment at the same point. Overall, this slight difference shows that the impact of the environment in the hilly Churchville cycle is greater than the impact of the efficiency over different times.

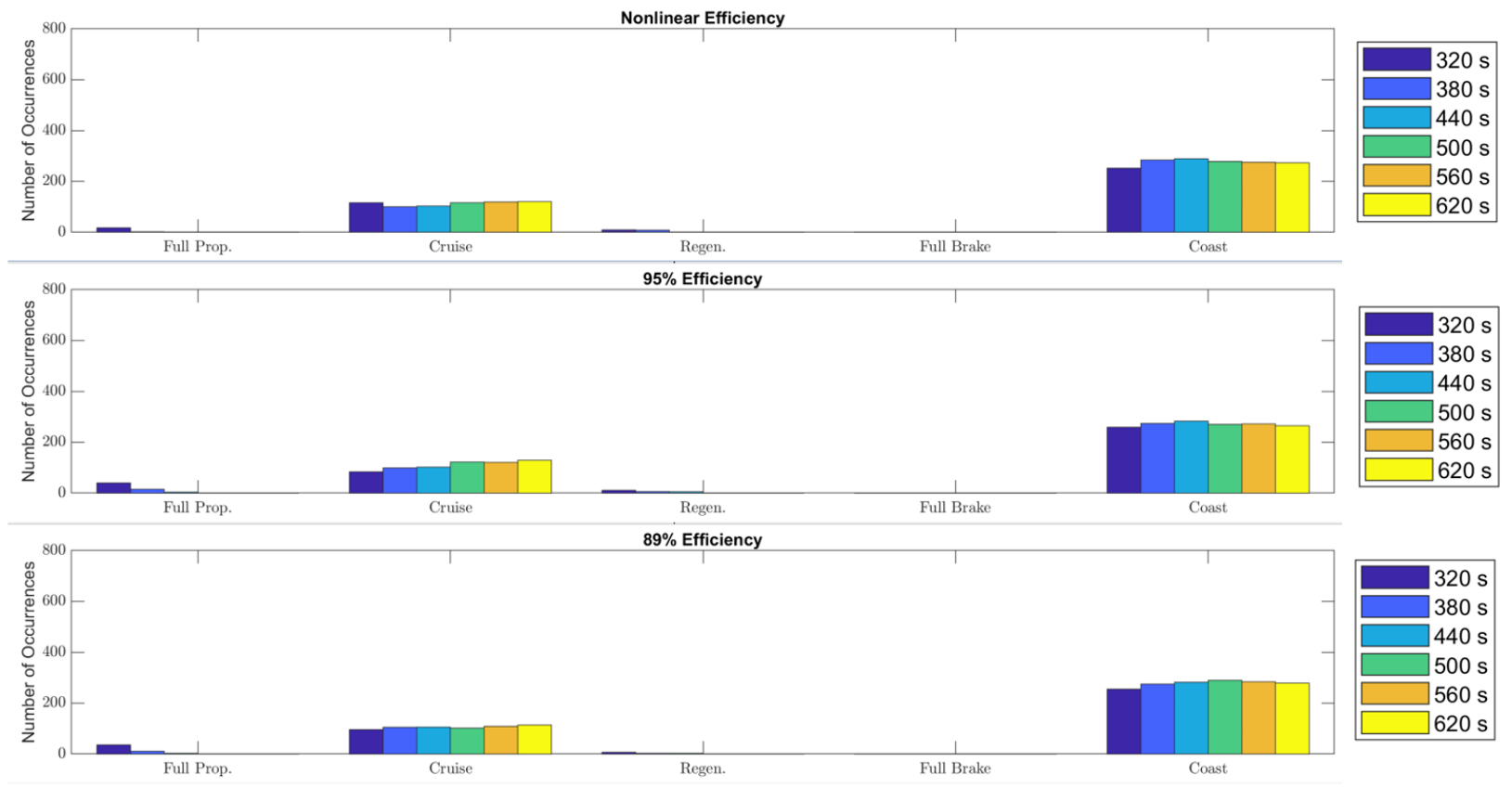

Figure 28 - Histogram Comparing Control Modes of Nonlinear Efficiency vs. Constant Efficiencies Over Time for Churchville B Cycle 


\section{Chapter 5: Conclusion and Future Work}

In this thesis work, the approach of synthesizing PMP analysis and Dynamic Programming has proved effective in computing the desired speed profile for energy minimization. The minimum energy consumption problem was formulated and analyzed by PMP. The analysis showed that only five modes are required to achieve the minimum energy consumption, which could significantly reduce computation time for DP. Then, the OCP was reformulated in the distance domain and solved by DP. For the considered vehicle, a light-weight military electrified vehicle, the simulation results demonstrate a tradeoff between energy consumption and trip time for both flat and hilly roads. For the flat Convoy cycle, the optimal speed cycle uses $21 \%$ less energy for the same trip duration or reduces the time by $14 \%$ with the same energy consumption while for the hilly Churchville B cycle, it uses $24 \%$ less energy or provides $24 \%$ reduction in time.

The impact of battery size, which dominates the regenerative braking capability, on energy consumption and control modes was also investigated. The benefit from increasing battery size, or regenerative power capability, depends on the operational environment. That is, for the non-hilly terrain, no significant reduction in energy consumption is observed regardless of the increase in regenerative power capability. On the other hand, for the hilly terrain, it is beneficial for the considered vehicle if larger batteries are installed; yet, no greater than $20 \mathrm{~kW}$. Furthermore, the sensitivity of energy consumption to regenerative-braking power limits and trip time is investigated. These studies provide important information that can be used in designing component size and scheduling operation to achieve the desired vehicle range. Furthermore, parametric studies of the influence of efficiency on the speed profile, controls modes, an energy consumption was 
conducted. The results showed that for the flat Convoy cycle, the motor efficiency did not change the operation significantly but was still able to slightly save energy throughout the trip. On the other hand, the Churchville B cycle was affected more evidently by the change in motor efficiency. The impact of the motor efficiency is more evident when the terrain contains more irregularities. Lastly, the work provided parametric studies that used nonlinear motor efficiency map to retrieve the results and compare them to the results retrieved from constant efficiency approach. The nonlinear motor efficiency was used in two different ways. Firstly, after DP computation of results using constant efficiency, the nonlinear efficiency map was used to compute energy consumption using the speed and forces retrieved. This suboptimal approach proved to be reliable and consistent. Nonetheless, another computation approach was made by using the nonlinear efficiency map throughout the DP computation. The results provide more reliable data as the speed, forces, and control modes retrieved by this approach are affected directly by the cost function. These approaches were directly compared to analyze the evident differences in speed profiles and control modes.

Future work includes co-optimization of speed profile and power management problems conducted to minimize total fuel consumption. The co-optimization problem will include battery state-of-charge dynamics and their constraints, in addition to nonlinear efficiency of the fuel cell system. 


\section{Appendix}

In this appendix, the remaining 20 control modes from the 27 possible ones discussed in Chapter 2 are shown. As discussed earlier, these control modes are infeasible due to the constraint that the vehicle only moves forward, and due to illogical contradiction, such as propulsion and braking occurring simultaneously.

$$
\begin{aligned}
& \frac{x_{2}}{\eta_{1}}+p_{2}<0, \quad x_{2} \eta_{2}+p_{2}<0, \quad p_{2}=0: u=\left[\overline{u_{1}}, 0, \text { undef }\right]^{\prime} \\
& \frac{x_{2}}{\eta_{1}}+p_{2}<0, \quad x_{2} \eta_{2}+p_{2}<0, \quad p_{2}>0: u=\left[\overline{u_{1}}, 0, \underline{u_{3}}\right]^{\prime} \\
& \frac{x_{2}}{\eta_{1}}+p_{2}<0, \quad x_{2} \eta_{2}+p_{2}=0, \quad p_{2}<0: u=\left[\overline{u_{1}}, \text { undef }, 0\right]^{\prime} \\
& \frac{x_{2}}{\eta_{1}}+p_{2}<0, \quad x_{2} \eta_{2}+p_{2}=0, \quad p_{2}=0: u=\left[\overline{u_{1}}, \text { undef }, \text { undef }\right]^{\prime} \\
& \frac{x_{2}}{\eta_{1}}+p_{2}<0, \quad x_{2} \eta_{2}+p_{2}=0, \quad p_{2}>0: u=\left[\overline{u_{1}}, \text { undef }, \underline{u_{3}}\right]^{\prime} \\
& \frac{x_{2}}{\eta_{1}}+p_{2}<0, \quad x_{2} \eta_{2}+p_{2}>0, \quad p_{2}=0: u=\left[\overline{u_{1}}, \underline{u_{2}}, \text { undef }\right]^{\prime} \\
& \frac{x_{2}}{\eta_{1}}+p_{2}<0, \quad x_{2} \eta_{2}+p_{2}>0, \quad p_{2}>0: u=\left[\overline{u_{1}}, \underline{u_{2}}, \underline{u_{3}}\right]^{\prime} \\
& \frac{x_{2}}{\eta_{1}}+p_{2}>0, \quad x_{2} \eta_{2}+p_{2}<0, \quad p_{2}=0: u=[0,0, \text { undef }]^{\prime} \\
& \frac{x_{2}}{\eta_{1}}+p_{2}>0, \quad x_{2} \eta_{2}+p_{2}<0, \quad p_{2}>0: u=\left[0,0, \underline{u_{3}}\right]^{\prime} \\
& \frac{x_{2}}{\eta_{1}}+p_{2}>0, \quad x_{2} \eta_{2}+p_{2}=0, \quad p_{2}=0: u=[0, \text { undef }, \text { undef }]^{\prime}
\end{aligned}
$$




$$
\begin{aligned}
& \frac{x_{2}}{\eta_{1}}+p_{2}>0, \quad x_{2} \eta_{2}+p_{2}=0, \quad p_{2}>0: u=\left[0, \text { undef, } \underline{u_{3}}\right]^{\prime} \\
& \frac{x_{2}}{\eta_{1}}+p_{2}>0, \quad x_{2} \eta_{2}+p_{2}>0, \quad p_{2}=0: u=\left[0, \underline{u_{2}}, \text { undef }\right]^{\prime} \\
& \frac{x_{2}}{\eta_{1}}+p_{2}=0, \quad x_{2} \eta_{2}+p_{2}<0, \quad p_{2}=0: u=[\text { undef }, 0, \text { undef }]^{\prime} \\
& \frac{x_{2}}{\eta_{1}}+p_{2}=0, \quad x_{2} \eta_{2}+p_{2}<0, \quad p_{2}>0: u=\left[\text { undef }, 0, \underline{u_{3}}\right]^{\prime} \\
& \frac{x_{2}}{\eta_{1}}+p_{2}=0, \quad x_{2} \eta_{2}+p_{2}=0, \quad p_{2}<0: u=[\text { undef, undef, }, 0]^{\prime} \\
& \frac{x_{2}}{\eta_{1}}+p_{2}=0, \quad x_{2} \eta_{2}+p_{2}=0, \quad p_{2}=0: u=[\text { undef, undef, undef }]^{\prime} \\
& \frac{x_{2}}{\eta_{1}}+p_{2}=0, \quad x_{2} \eta_{2}+p_{2}=0, \quad p_{2}>0: u=\left[\text { undef }, \text { undef }, \underline{u_{3}}\right]^{\prime} \\
& \frac{x_{2}}{\eta_{1}}+p_{2}=0, \quad x_{2} \eta_{2}+p_{2}>0, \quad p_{2}<0: u=\left[\text { undef, } \underline{u_{2}}, 0\right]^{\prime} \\
& \frac{x_{2}}{\eta_{1}}+p_{2}=0, \quad x_{2} \eta_{2}+p_{2}>0, \quad p_{2}=0: u=\left[\text { undef }, \underline{u_{2}}, \text { undef }\right]^{\prime} \\
& \frac{x_{2}}{\eta_{1}}+p_{2}=0, \quad x_{2} \eta_{2}+p_{2}>0, \quad p_{2}>0: u=\left[\text { undef }, \underline{u_{2}}, \underline{u_{3}}\right]^{\prime}
\end{aligned}
$$




\section{References}

[1] F. Ding and H. Jin. "On the optimal speed profile for eco-driving on curved roads." IEEE Transactions on Intelligent Transportation Systems, pages 1-11, 2018.

[2] Union of Concerned Scientists. Smart policy can improve fuel efficiency. https://www.ucsusa.org/clean-vehicles/fuel-efficiency.WysXm6dKhPY.

[3] S. Singh, "Global Electric Vehicle Market Looks To Power Up In 2018," Forbes, 14-Apr2018. [Online]. Available: https://www.forbes.com/sites/sarwantsingh/2018/04/03/globalelectric-vehicle-market-looks-to-fire-on-all-motors-in-2018/\#66ea60132927.

[4] Global Plug-in Sales for Q1-2018 the electric vehicle world sales database, 2018. www.evvolumes.com/country/total-world-plug-in-vehicle-volumes/.

[5] A.B. Schwarzkopf and R.B. Leipnik. Control of highway vehicles for minimum fuel consumption over varying terrain. Transportation Research, 11(4): 279 - 286, 1977.

[6] Thorpe, C., Hebert, M., Kanade, T., and Shafer, S.,1989. "Vision and Navigation: The Carnegie Mellon Navlab". In High Precision Navigation, K. Linkwitzand U. Hangleiter, eds., Springer Berlin Heidelberg, pp. 97-122.

[7] Vclav Kratochvl and Ji Vomlel. "Influence diagrams for speed profile optimization." International Journal of Approximate Reasoning, 88:567 - 586, 2017.

[8] Baichuan Mo, Ruimin Li, and Xianyuan Zhan. Speed profile estimation using license plate recognition data. Transportation Research Part C: Emerging Technologies, 82:358 - 378, 2017.

[9] M. A. S. Kamal, T. Hayakawa, and J. i. Imura. Road-speed profile for enhanced perception of traffic conditions in a partially connected vehicle environment. IEEE Transactions on Vehicular Technology, pages 1-1, 2018.

[10] Amrani, A., Ben Hamida A., Liu T., and Langlois O. "Train speed profiles optimization using a genetic algorithm based on a random-forest model to estimate energy consumption". In Transport Research Arena (TRA) 2018, Vienne, Austria, April 2018.

[11] Shoichiro W., Takafumi K., Yoshichika N., and Masafumi M. "Optimized energy saving speed profile in linear motor railway system". Electrical Engineering in Japan, 202(3): 2232.

[12] Yang S., Wu J., Yang X., Sun H., and Gao Z. "Energy-efficient timetable and speed profile optimization with multi-phase speed limits: Theoretical analysis and application". Applied Mathematical Modelling, 56:32 - 50, 2018.

[13] Michael J. Ogburn, Douglas J. Nelson, Keith Wipke, and Tony Markel. Modeling and validation of a fuel cell hybrid vehicle. In Future Car Congress. SAE International, apr 2000. https://doi.org/10.4271/2000-01-1566.

[14] U.S Department of Energy. Fuel cell electric vehicles, 2018. https://www.afdc.energy.gov/vehicles/fuelcell.html.

[15] B. Lu, B. Natarajan, and N. Schulz. "Optimal control-based power management in hybrid military vehicle". In 2012 IEEE International Electric Vehicle Conference, pages 1-7, March 2012. 
[16] R.D. Geertsma, R.R. Negenborn, K. Visser, and J.J. Hopman. "Design and control of hybrid power and propulsion systems for smart ships: A review of developments". Applied Energy,194:30 - 54, 2017.

[17] E. I. Grøtli and T. A. Johansen, "Path planning for UAVs under communication constraints using SPLAT! and MILP," Journal of Intelligent and Robotic Systems, vol. 65, no. 1-4, pp. 265-282, 2012.

[18] Wang, Q., and Chang, X. (2016). "The Optimal Trajectory Planning for UAV in UAV-aided Networks." Cloud Computing and Security Lecture Notes in Computer Science, 192-204.

[19] J. S. Bellingham, A. G. Richards and J. P. How, "Receding Horizon Control of Autonomous Aerial Vehicles", ACC, Anchorage, AK, May 2002.

[20] V. Nezhadali, L. Eriksson, "Wheel loader optimal transients in the short loading cycle", Proc. 19th IFAC World Congr., pp. 7917-7922, 2014.

[21] D. Chang, E. Morlok, "Vehicle speed profiles to minimize work and fuel consumption", J. Transp. Eng., vol. 131, no. 3, pp. 173-182, 2005.

[22] L. Nouveliere, M. Braci, L. Menhour, H. T. Luu, S. Mammar, "Fuel consumption optimization for a city bus", Proc. UKACC Control Conf., 2008.

[23] A. Sciarretta, L. Guzzella, J. van Baalen, "Fuel optimal trajectories of a fuel cell vehicle", Proc. IFAC Int. Conf. Advances Vehicle Control and Safety, 2004.

[24] J.-J. Santin, The World's Most Fuel-Efficient Vehicle: Design and Development of Pac-Car II, vdf Hochschulverlag AG an der ETH Zurich, 2007.

[25] Sciarretta, A., De Nunzio, G., and Ojeda, L. L., 2015. "Optimal eco-driving control: Energy-efficient driving of road vehicles as an optimal control problem”. IEEE Control Systems, 35(5), pp. 71-90.

[26] F. Lattemann, K. Neiss, S. Terwen, T. Connolly, "The Predictive Cruise Control—A system to reduce fuel consumption of heavy duty trucks".

[27] E. Hellström, M. Ivarsson, J. Aslund, L. Nielsen, "Look-ahead control for heavy trucks to minimize trip time and fuel consumption", Contr. Eng. Pract., vol. 17, no. 2, pp. 245-254, 2009.

[28] B. Passenberg, P. Kock, O. Stursberg, "Combined time and fuel optimal driving of trucks based on a hybrid model", Proc. 10th European Control Conf., pp. 4955-4960, 2009.

[29] T. van Keulen, B. de Jager, D. Foster, M. Steinbuch, "Velocity trajectory optimization in hybrid electric trucks", Proc. American Control Conf., pp. 5074-5079, 2010.

[30] G. Heppeler, M. Sonntag, O. Sawodny, "Fuel efficiency analysis for simultaneous optimization of the velocity trajectory and the energy management in hybrid electric vehicles", Proc. 19th IFAC World Congr., pp. 6612-6617, 2014.

[31] E. Ozatay, U. Ozguner, J. Michelini, D. Filev, "Analytical solution to the minimum energy consumption based velocity profile optimization problem with variable road grade", Proc. 19th IFAC World Congress, 2014.

[32] E. Hellström, J. Slund, and L. Nielsen. Design of an efficient algorithm for fuel-optimal look-ahead control. Control Engineering Practice, 18(11): 1318 - 1327, 2010. Special Issue on Automotive Control Applications, 2008 IFAC World Congress.

[33] Z. Li, L.Chen, C.Roberts, and N.Zhao, 2018. "Dynamic trajectory optimization design for railway driver advisory system". IEEE Intelligent Transportation Systems Magazine, 10(1), Spring, pp. 121-132. 
[34] Dib, W., Chasse, A., Moulin, P., Sciarretta, A., and Corde, G., 2014. “Optimal energy management for an electric vehicle in eco-driving applications". Control Engineering Practice, 29, pp. 299-307.

[35] Hu, J., Shao, Y., Sun, Z., Wang, M., Bared, J., and Huang, P., 2016. "Integrated optimal eco-driving on rolling terrain for hybrid electric vehicle with vehicle-infrastructure communication". Transportation Research Part C: Emerging Technologies, 68, pp. 228244.

[36] Khmelnitsky, E., 2000. “On an optimal control problem of train operation”. IEEE Transactions on AutomaticControl,45(7), Jul, pp. 1257-1266.

[37] Liu, R. R., and Golovitcher, I. M., 2003. "Energy-efficient operation of rail vehicles". Transportation Research Part A: Policy and Practice, 37(10), pp. 917 -932.

[38] Howlett, P., Pudney, P., and Vu, X., 2009. "Local energy minimization in optimal train control". Automatica, 45(11), pp. $2692-2698$.

[39] Albrecht, A. R., Howlett, P. G., Pudney, P. J., and Vu,X., 2013. "Energy-efficient train control: From local convexity to global optimization and uniqueness". Automatica, 49(10), pp. $3072-3078$.

[40] Maamria, D., Gillet, K., Colin, G., Chamaillard, Y., and Nouillant, C., 2017. "Optimal ecodriving for conventional vehicles: Simulation and experiment”. Vol. 50, pp. 12557 12562. 20th IFAC World Congress.

[41] Miyatake, M., and Matsuda, K. "Energy saving speed and charge/discharge control of a railway vehicle with on board energy storage by means of an optimization model". IEEJ Transactions on Electrical and Electronic Engineering, 4(6), pp. 771-778.

[42] Bocharnikov, Y., Tobias, A., and Roberts, C., 2010."Reduction of train and net energy consumption using genetic algorithms for trajectory optimization”. pp. 1-5.

[43] Nikovski, D., Lidicky, B., Zhang, W., Kataoka, K., and Yoshimoto, K., 2012. "Markov decision processes for train run curve optimization”. In 2012 Electrical Systems for Aircraft, Railway and Ship Propulsion, pp. 1-6.

[44] Lu, S., Hillmansen, S., Ho, T. K., and Roberts, C.,2013. "Single-train trajectory optimization”. IEEE Transactions on Intelligent Transportation Systems, 14(2), June, pp. 743-750.

[45] Xu, J., Nikovski, D., and Kimura, S., 2015. “A frame-work for real-time near-optimal train run-curve computation with dynamic travel time and speed limits". In 2015 American Control Conference (ACC), pp. 533-540.

[46] Petit, N., and Sciarretta, A., 2011. "Optimal drive of electric vehicles using an inversionbased trajectory generation approach". IFAC Proceedings Volumes, 44(1), pp. 14519 14526. 18th IFAC World Congress.

[47] Mensing, F., Trigui, R., and Bideaux, E., 2011. "Vehicle trajectory optimization for application in eco-driving". In 2011 IEEE Vehicle Power and Propulsion Conference, pp. 16.

[48] Mensing, F., Bideaux, E., Trigui, R., Ribet, J., and Jeanneret, B., 2014. "Eco-driving: An economic or eco-logic driving style?". Transportation Research Part C: Emerging Technologies, 38, pp. $110-121$.

[49] Chen, D., Kim, Y., and Stefanopoulou, A. G., 2018. "Soc node planning for PHEVs with segmented traffic information". In 2018 American Control Conference. 
[50] Chen, Y., Li, X., Wiet, C., and Wang, J., 2014. "Energy management and driving strategy for in-wheel motor electric ground vehicles with terrain profile preview". IEEE Transactions on Industrial Informatics, 10(3), Aug, pp. 1938-1947.

[51] He, X., Liu, H. X., and Liu, X., 2015. "Optimal vehicle speed trajectory on a signalized arterial with consideration of queue". Transportation Research Part C: Emerging Technologies, 61, pp. $106-120$.

[52] Zeng, X., and Wang, J., 2018. "Globally energy-optimal speed planning for road vehicles on a given route". Transportation Research Part C: Emerging Technologies,93, pp. 148 - 160.

[53] Ken-Jui Tsao, Si-Sheng Wan, Po-Ting Kuo, and Ran-Ren Chang. Trajectory generation for vehicle moving with constraints on a complex terrain. In Proceedings of 2003 IEEE International Conference on Robotics and Automation, 2003.

[54] Kim, N., Cha, S., and Peng, H., 2011. "Optimal control of hybrid electric vehicles based on Pontryagin's Minimum Principle". IEEE Transactions on Control Systems Technology, 19(5), Sept, pp. 1279-1287.

[55] Ahmadizadeh, P., Mashadi, B., and Lodaya, D., 2017.“Energy management of a dual-mode power-split powertrain based on the pontryagin's minimum principle". IET Intelligent Transport Systems, 11(9), pp. 561-571.

[56] Kelley, H. J., 1964. "A second variation test for singular extremals". AIAA Journal,2, Aug., pp. 1380-1382.

[57] Bellman, Richard (1954), "The theory of dynamic programming", Bulletin of the American Mathematical Society 60: 503-516, doi:10.1090/S0002-9904-1954-09848-8, MR0067459.

[58] Sniedovich, M. (2010), Dynamic Programming: Foundations and Principles, Taylor \& Francis, ISBN 9780824740993

[59] Atkeson, C. G. "Randomly Sampling Actions In Dynamic Programming," 2007 IEEE International Symposium on Approximate Dynamic Programming and Reinforcement Learning, 2007.

[60] Bellman, Richard (1957), Dynamic Programming, Princeton University Press. Dover paperback edition (2003), ISBN 0486428095.

[61] Kirk, D. E (1970). Optimal Control Theory: An Introduction. Englewood Cliffs, NJ: Prentice-Hall. p. 86-90. ISBN 0-13-638098-0.

[62] Kamien, M. I.; Schwartz, N. L. (1991). Dynamic Optimization: The Calculus of Variations and Optimal Control in Economics and Management (Second ed.). New York: Elsevier. p. 261. ISBN 978-0-444-01609-6.

[63] Elbert, P., Ebbesen, S., and Guzzella, L., 2013. "Implementation of dynamic programming for n-dimensional optimal control problems with final state constraints". IEEE Transactions on Control Systems Technology, 21(3), May, pp. 924-931.

[64] "Military and Veteran Benefits, News, Military.com. [Online]. Available: https://www.military.com/defensetech/2018/02/01/army-test-first-robotic-combat-vehicle2021.html.

[65] Sebeck, K., Mange, J., MacLennan, J., and Rizzo, D.,2017. “Characterization of army ground vehicle drive cycles". In NDIA Ground Vehicle Systems Engineering and Technology Symposium.

[66] WANG, Jun-chang \& Bin, Wang \& DAI, Yi-fan \& HAN, Zong-qi. (2017). Research on the Strategy of Gliding in Eco-driving. DEStech Transactions on Environment, Energy and Earth Science. 10.12783/dteees/icesee2017/7875. 
[67] E.S. Li, Y. Wang, W.M. Wu, "Research of Correct Use and Saving Energy of Automobile," Tractor \& Farm Transporter. vol. 37, pp.111-115, March 2010.

[68] S. Xu, S.E. Li, X. Zhang, B. Cheng, "Fuel-optimal cruising strategy for road vehicles with step-gear mechanical transmission," IEEE Transactions on Intelligent Transportation Systems. vol. 16, pp. 1-12, June 2015. 